\section{Automatic Radar Antenna Scan
Type Recognition in Electronic Warfare}

\author{
BILLUR BARSHAN \\ BAHAEDDIN ERAVCI \\ Bilkent University
}

We propose a novel and robust algorithm for antenna scan type (AST) recognition in electronic warfare (EW). The stages of the algorithm are scan period estimation, preprocessing (normalization, resampling, averaging), feature extraction, and classification. Naive Bayes (NB), decision-tree (DT), artificial neural network (ANN), and support vector machine (SVM) classifiers are used to classify five different ASTs in simulation and real experiments. Classifiers are compared based on their accuracy, noise robustness, and computational complexity. DT classifiers are found to outperform the others.

Manuscript received August 20, 2010; revised May 1, 2011; released for publication October 4, 2011.

IEEE Log No. T-AES/48/4/944181.

Refereeing of this contribution was handled by R. Narayanan.

Authors' address: Department of Electrical and Electronics Engineering, Bilkent University, Bilkent, Ankara, TR-06800, Turkey, E-mail: billur@ee.bilkent.edu.tr).

0018-9251/12/\$26.00 (c) 2012 IEEE
LIST OF ABBREVIATIONS

CW Continuous-wave

EW Electronic warfare

EM Electromagnetic

LFM Linear frequency modulation

DTW Dynamic time warping

PA Pulse amplitude

PW Pulsewidth

DoA Direction of arrival

ToA Time of arrival

PRI Pulse repetition interval

ASPS Antenna scan pattern simulator

AST Antenna scan type

ASP Antenna scan period

ASR Antenna scan rate

FFT Fast Fourier transform

DFT Discrete Fourier transform

DCT Discrete cosine transform

DWT Discrete wavelet transform

NB Naive Bayes

DT Decision tree

ANN Artificial neural networks

SVM Support vector machine

WEKA Waikato Environment for Knowledge Analysis

CART Classification and regression trees

BFTree Best first tree

GUI Graphical user interface

SNR Signal-to-noise ratio

\section{INTRODUCTION}

Military operations are executed in an information environment where the electromagnetic (EM) spectrum is becoming increasingly more complex. EM devices are being used in a stand-alone capacity and in networks by both civilian and military organizations and by individuals for intelligence, communication, navigation, sensing, information storage and processing, as well as for a variety of other purposes [1]. Vulnerability in this relatively new dimension of warfare could mean a battle may be lost without even making physical contact with hostile units.

Different technologies and military doctrines have evolved as the use of the EM spectrum has expanded vastly in many different bands. Electronic warfare (EW) is an umbrella term used to define any activity that can control the spectrum, attack an enemy, or impede enemy assaults via the spectrum. The goal of EW is to deny the opponent the advantage of, and ensure friendly unimpeded access to, the EM spectrum $[2,3]$. Signal intelligence (SIGINT) missions are employed on a daily basis in peace time to support EW operations during war time. Such missions are responsible for recording, analyzing, and forming parameter databases of EM emissions of particular importance. 
In this paper we address the problem of radar antenna scan type (AST) recognition and propose an algorithm to estimate the radar antenna scan period (ASP) followed by AST classification. Both ASP and AST are vital for EW systems in emitter classification and for the timing of electronic counter measures [4]. A change of AST is also crucial in determining threat levels from radar. Despite their importance, to the best of our knowledge, there is a paucity of studies in the open literature on automatic AST recognition because of the classified nature of EW work. The conventional solution to the problem in EW is to employ a human operator who listens to the radar tone generated by the received pulses. The operator guesses the AST and estimates its period with a stopwatch. It is evident, then, that this is an expensive solution and that its robustness and accuracy depends on the experience level of the operator. The main contribution of this paper is the automation of this process through the development of a novel ASP estimation and AST classification technique based on signal warping (resampling) and pattern recognition. Automating the AST recognition process completely eliminates the need for a human EW operator. It can be applied to both modulated and unmodulated radar signals. The algorithm is sufficiently general and robust that it can also be employed effectively to classify signals with varying periodicities in other application areas.

The only related works are two patents that have been issued in the United States [5]. The first patent, issued in 1980, describes a scan pattern generator probably used in EW simulators. In 2004, another patent was issued to a Lockheed Martin employee for a system that automatically recognizes ASTs [6]. In this patent, Laplace transform and the fast Fourier transform (FFT) implementation of the discrete Fourier transform (DFT) are used. The basic ASTs are determined and sample data from each type are saved in a database. The newly recorded signal is transformed to the frequency domain and correlated with the previously saved samples. The type that results in the maximum correlation is determined to be the AST of the radar. A closer look into this patent shows that most parts of the process are vague and the remaining transparent part is quite trivial. Changes in the ASP and the variation of other parameters such as antenna beamwidth are probably neglected and the algorithm looks far from robust. The algorithm proposed in this paper for AST classification is not based on correlation techniques but, rather, based on pattern recognition. It also takes into account and handles the variability of the ASP for different radars and warps (resamples) each signal so that each period is represented by a fixed number of samples.

Despite the lack of work on AST recognition, studies exist in the analysis of time series from which valuable insights can be acquired. Time series analysis finds many applications in science, engineering, medicine, economics, and finance. Most studies have compared time series based on their Euclidean distance or its variations to detect similarities. However, Euclidean distance is a very brittle distance measure that can be highly sensitive to outliers. A more robust distance or similarity measure for time series is based on dynamic time warping (DTW) that uses linear programming to find the best possible match between time series even if they are out of phase in the time axis [7]. Because of its flexibility, DTW is widely used in many applications. One example is vowel classification in the speech processing area [8]. Everyone forms vowels of similar shape but the length and frequency vary from person to person. If both the shape of the signal and its length change within the same class, DTW fails to find a match. In our problem, both the signal length (period) and its shape change (because of the position of the radar and the EW receiver) within one class of data, so that the use of DTW becomes unsuitable. Other measures of similarity are based on autocorrelation functions, characteristic points, genetic algorithms, artificial neural networks (ANNs), Markov models, etc. In [9] a survey on time series data mining is provided and 11 similarity measures are experimentally compared in terms of their error rates. Similarities of time series are sometimes measured by transforming the data to other domains using transformations such as DFT, discrete wavelet transform (DWT), Karhunen-Loève transform, singular value decomposition, or principal component analysis [10-13].

Some recent works address the use of the ASP for emitter localization. Scan-based emitter localization is a passive geolocation technique for stationary pulsed radars. It takes advantage of the geometric constraints introduced by the uniform rotational motion of the antenna main beam as it sweeps across a number of separate receivers at different locations. The interception times of the emitter are used to define time difference of arrival-like equations that are solved using a least-squares error estimator. Knowledge of the radar scan rate (or sweep rate) and radar beam intercept times are utilized to determine the subtended angles of the radar location from receiver pairs. A collection of subtended angles is then used to estimate the radar location. The geolocation approach greatly relaxes the precise synchronization need between the receiver stations and makes geolocation possible with parameters (pulse amplitude sequence) that can be conveniently measured [14]. In [15] a maximum likelihood scan-based localization algorithm is presented and the Cramér-Rao lower bound is derived for the emitter location estimate. Based on maximization of the determinant of the Fisher information matrix, online gradient-based receiver trajectory optimization algorithms are developed. In [16] a joint estimator for the scan rate and the position 
of a scanning emitter is proposed based on nonlinear least-squares estimation.

The rest of this paper is organized as follows. Section II provides fundamental information about pulsed radar systems and their distinctive parameters. The primary focus is on AST and ASP. The basic ASTs are overviewed in Section III. Section IV briefly describes the antenna scan pattern simulator coded to synthesize radar signals. Section V explains the proposed algorithm in detail. The algorithm is validated with synthetic and real data and a comparison between four AST classifiers is presented. Section VI concludes the paper and provides some possible future research directions.

\section{PULSED RADAR PARAMETERS AND ELECTRONIC WARFARE}

The type of radar considered in this study is conventional pulsed radar widely used in military applications for searching, detecting, and tracking airborne targets [17-19]. Accurate tracking is crucial for following a particular target (such as an aircraft) or an unresolved cluster of targets (such as an aircraft formation) as well as for efficient use of weapons against the target. Over the years, different types of volume search and target-tracking methods have evolved. These methods, usually periodic, are deployed in the various radar from different manufacturers with widely varying parameters [4]. The parameters that characterize a pulsed radar are its carrier frequency, bandwidth, pulsewidth (PW), pulse amplitude (PA), time and direction of arrival (ToA and DoA), pulse repetition interval (PRI), signal power, lobe duration, AST, and ASP [20]. The types of intrapulse modulation and PRI modulation used (if any) are also very important in characterizing a radar. The bandwidth of the radar depends on the PW as well as the intrapulse modulation and determines the range resolution of a radar. Modern radars usually have multiple signal bandwidths. Radars utilize different kinds of PRI patterns such as constant, stagger, and agile PRI, for which the PRI is constant, deterministic, and random, respectively [18].

In pulsed radar systems, the carrier is an RF signal, typically of microwave frequencies, which is usually (but not always) modulated. Most simple ranging radars use pulse modulation, with or without other supplementary modulation, where the carrier is simply switched on and off in synchronization with the pulses. Radar systems that use modulation within the pulse (intrapulse modulation) are referred to as pulse compression radar systems. Pulse compression allows a radar to utilize a long pulse to achieve high radiated energy, but at the same time benefit from the fine range resolution of a short pulse. This is accomplished by employing frequency or phase modulation to widen the signal bandwidth. (Amplitude modulation is also possible but is rarely used.) This allows the long pulse to be compressed in the receiver by an amount equal to the reciprocal of the signal bandwidth. Linear frequency modulation (LFM) and phase-coded pulse are the most widely applied modulation types used in modern radars to obtain fine range resolution. The LFM pulse (or chirp) with a sweeping frequency has the advantage of greater bandwidth while keeping the pulse duration short and envelope constant. In phase coding, where the phase of the carrier wave is altered according to some specific pattern, binary codes (e.g., Barker codes), random codes, or alternating codes can be used. Other pulse-compression methods include nonlinear FM, discrete frequency shift, polyphase codes, compound Barker codes, code sequencing, complementary codes, pulse burst, and stretch [18].

Antennas are the crucial and indispensable parts of radar systems as they radiate and receive EM waves. Radar systems use a wide variety of antenna types, specialized for different applications and functionalities [21]. Since the coverage of the antenna beamwidth in azimuth and elevation is usually not sufficient for the radar's requirements, the antenna is steered, either electronically or mechanically, to the desired part of the space [4]. Considering a hemispherical volume to be covered, the number of distinct steering positions for a mechanically-steered antenna is given by $2 \pi / \Delta \theta \Delta \phi$, where $\Delta \theta$ and $\Delta \phi$ are the azimuth and elevation beamwidths, respectively. This formula is not valid for electronically-scanned planar phased arrays since the beam broadens in angle (although it remains invariant in sine space).

The EW receiver tries to acquire information about radar in the environment (and possibly jam them) to protect the platform on which it is located while the platform performs its mission. In systems that detect ToA, PA, and duration, many options for antenna scan analysis are available. Time-domain analysis techniques are more useful and are usually based on heuristics rather than theory [4]. The approach we have taken in this work for scan analysis is based on measuring the PA and estimating the ASP in the time domain in order to determine the AST.

PA is the received signal power of the pulse at the EW antenna and is given by

$$
P_{r}(t)=\frac{P_{t} G_{r} \lambda^{2}}{(4 \pi R)^{2} L} G_{t}[\theta(t), \phi(t)]
$$

where $P_{t}$ and $P_{r}$ are the transmitted and the received power, $G_{r}$ is the receiver antenna gain, $\lambda$ is the wavelength, $R$ is the range between the radar and the EW receiver, and $L$ is the loss factor. Atmospheric propagation losses are proportional to range and frequency and can be significant for low elevation angles which are commonly encountered. Polarization 
TABLE I

The Importance of Various Emitter Parameters in the Processing of Radar Signals [20]

\begin{tabular}{c|c|ccc}
\hline \hline \multirow{2}{*}{ Parameter } & Pulse Train De-Interleaving & Emitter Identification & Emitter Localization \\
\hline measured & frequency & very useful & very useful & some use \\
& ToA & not useful & not useful & very useful \\
& AoA & very useful & not useful & very useful \\
& PW & very useful & some use & nseful \\
& PA & some use & very useful use & some use \\
& PRI & very useful & not useful \\
& PRI type & very useful & very useful & some use \\
& AST and ASP & not useful & very useful useful \\
\hline
\end{tabular}

mismatch between the two antennas is another factor that affects the PA. As the antenna rotates to different parts of the volume, the received power changes according to the gain of the antenna at the angular position of the EW receiver. Hence, $G_{t}[\theta(t), \phi(t)]$ is the radar transmitter antenna gain at the azimuth and elevation angles where the EW receiver is located at time $t$. The term $P_{t} G_{r} \lambda^{2} /(4 \pi R)^{2} L$ is assumed to be constant because the geometry (range and angle) between the radar and the EW receiver is assumed to be changing negligibly. This assumption is valid for stationary engagement scenarios and scenarios where the scan rate of the antenna is much faster than the motion of the system platform, which is mostly the case in EW. When the relative motion between the radar and the EW receiver is significant, this assumption is no longer valid, the range $R$ becomes time dependent, and the received signal power in (1) becomes a function of the changing $R$. Assuming that it is possible to constantly update the positions of the radar and the EW receiver through the use of geolocation, the range $R$ can be recalculated at each scan and used in (1). It is also possible to calibrate the radar system to measure velocity together with range to exploit Doppler information.

If the received power is above the sensitivity level of the EW receiver, radar pulses with different amplitudes are detected and analyzed. The sensitivity level of the receiver depends on its bandwidth and noise figure. The ASP is the shortest time interval between the repetitive patterns observed in a PA recording. Instead of the ASP, sometimes the antenna scan rate (ASR) is used, which is simply the reciprocal of the ASP. When the ASP is short so that the ASR is large (as is the case for conical scans for example), the latter is stated more often because of the convenience of numerical representation.

The parameters that can be measured by an EW receiver depend on its complexity and capabilities. The received pulses or pulse trains are processed by signal sorting or de-interleaving algorithms that classify and associate them with different radar emitters [22]. Although all of the parameters mentioned at the beginning of this section can be used in the de-interleaving process, they are not equally beneficial in discriminating emitters and their computational costs are not the same. The importance of the different emitter parameters in radar signal processing is summarized in Table I where it is stated that AST and ASP of the emitter are very useful parameters in emitter identification [20].

\section{BASIC ANTENNA SCAN TYPES}

Radars use different search-and-track strategies to cover the specific region they are directed to. These strategies determine the radar's AST. The basic ASTs are described in [4], [23], [24] and are summarized below.

1) Circular Scan: The circular scan is widely used in search radars. The radar scans the complete azimuth plane $\left(360^{\circ}\right)$ at a constant angular speed (Fig. 1). The antenna typically has a large elevation beamwidth, called a fan beam, to cover the whole elevation space without having to scan it. The EW receiver samples the PA with a sampling period equal to the PRI since the pulses are received with PRI intervals in time. The ASP has quite a large range, on the order of 1-10 s.

2) Sector Scan: In the sector scan, the radar sweeps a specific angular sector back and forth at constant angular speed (Fig. 2(a)). The EW antenna receives periodic and symmetric main beams, as shown in Fig. 2(b). Two main beams with equal peaks are expected for each full period. The ASP is on the order of seconds.

3) Raster Scan: The two scan types described above search only in azimuth, but the raster scan searches both in azimuth and elevation (Fig. 3). The radar scans a specific angular sector in azimuth and increments its elevation after completing the sector, similar to the raster scan on TV screens. There can be several elevation levels. Figure 3 shows the antenna motion and the received PA as a function of ToA. The period is constant and in each full period, a main beam is intercepted for each bar of the raster scan. However, since the elevation is also changing in this scan type, the received PA varies with the elevation of the EW receiver. 


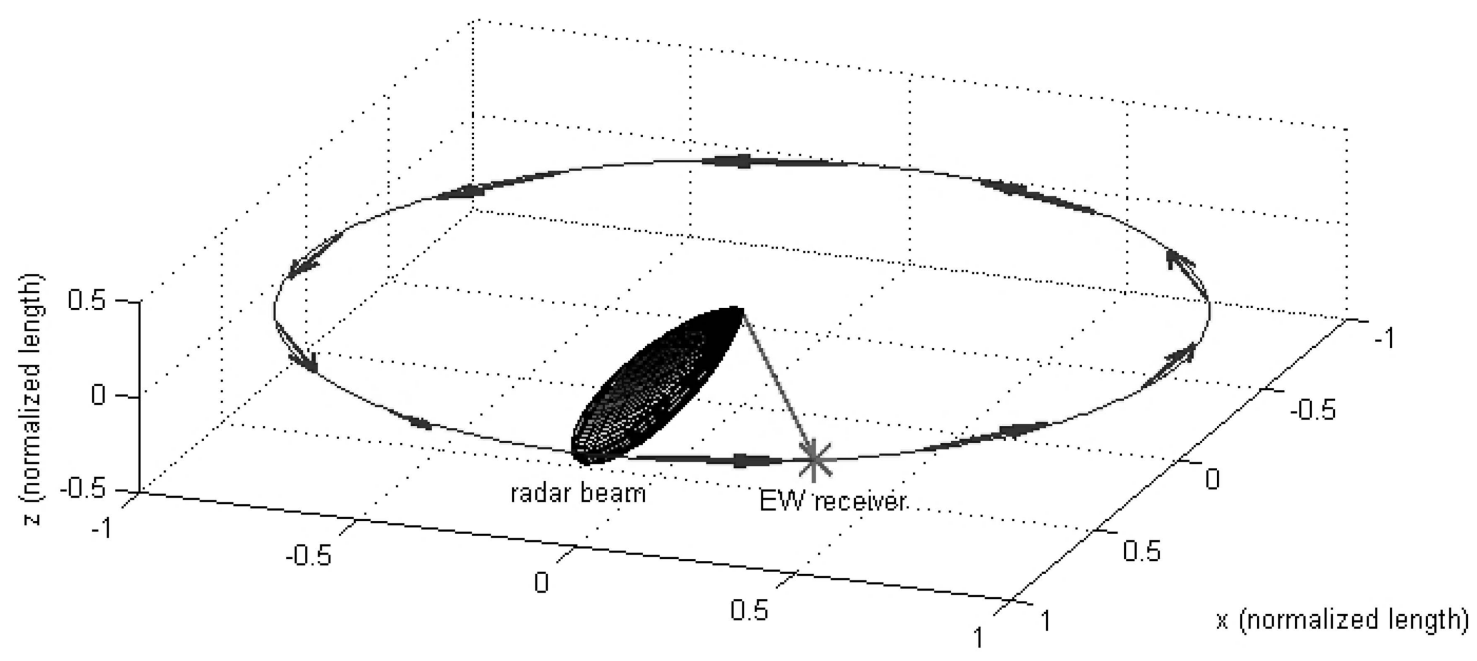

$y$ (nomalized length)

(a)

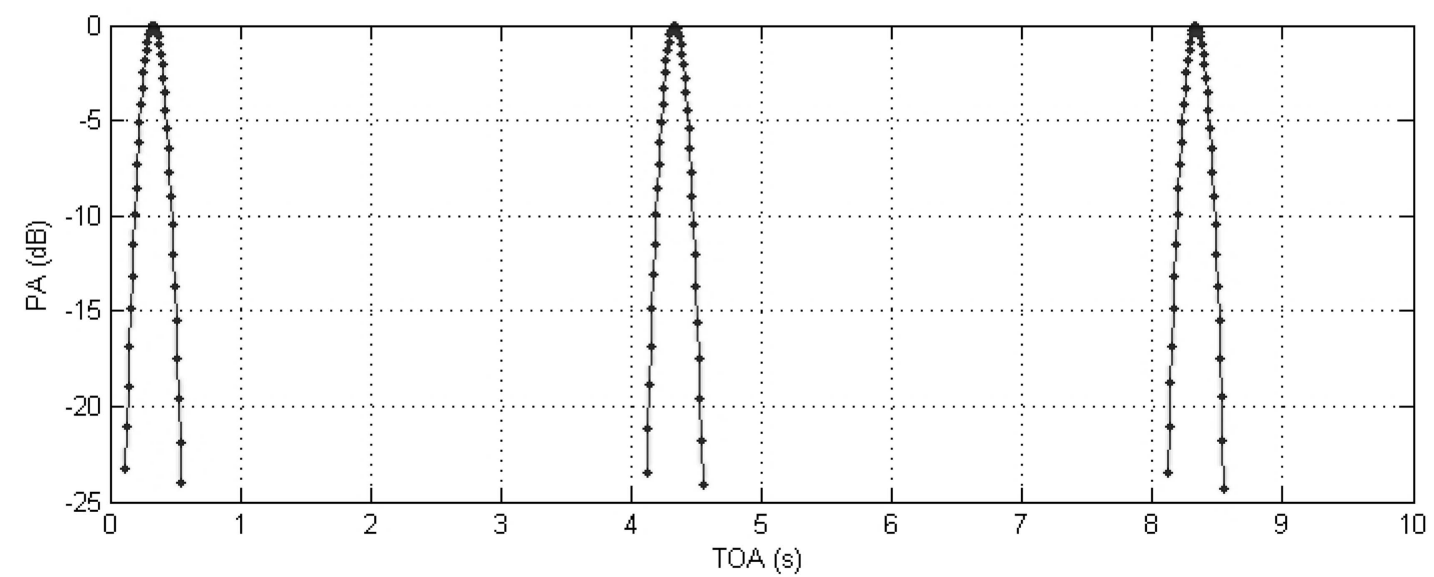

(b)

Fig. 1. Circular scan. (a) Main beam positions. (b) PA versus ToA graph, where solid dots indicate measured PAs.

4) Helical Scan: In the helical scan, the radar revolves $360^{\circ}$ several times while the elevation changes continuously so that the radar scans a specific sector in elevation. After a complete scan period, the elevation is set back to where the scan began. The shape of the scan resembles a helix (Fig. 4). The received PA versus ToA signal (Fig. 4(b)), is similar to the circular scan except that the peaks of the pattern change because of the motion in the elevation plane.

5) Conical Scan: A critical piece of information in scanning is whether radar is tracking the platform the EW receiver is located on. A conical scan indicates that radar is trying to lock onto the platform by scanning conically around it to place it on the $3 \mathrm{~dB}$ beamwidth. Figure 5(a) illustrates the movement of the antenna. The received signal, illustrated in Fig. 5(b), is initially a sinusoid because the beam travels closer to, then farther from the EW receiver. As the lock improves, the amplitude of the sinusoid decreases. The period of the sinusoid is distinct for each type of radar. Eventually, when the radar is locked completely, the track process converges to a perfect track. In other words, when the platform is right on the $3 \mathrm{~dB}$ beamwidth, the received PA becomes constant. The complete lock of this scan type is sometimes called a fixed scan. The PA during the locking-on process is illustrated in Fig. 5(c).

Other antenna scan types are bow tie scan and Archimedes spiral type scan, which are not as common as those described above. These are not considered in this study.

Unlike radars that have basic preprogrammed scan types, as those considered in this paper, phased-array radars have very complex and dynamic beam management algorithms $[4,25]$. A phased array may be used to generate a fixed radiation pattern, or to scan rapidly in azimuth or elevation. Modern phased-array radars rely on digital beamforming and employ track-while-scan algorithms that dynamically change the beam formation of the radar according to the received pulses, threat location and speed, etc. Therefore, the beam shape of phased-array radars change with the steering angle. The beamwidth is approximately inversely proportional to the angle 


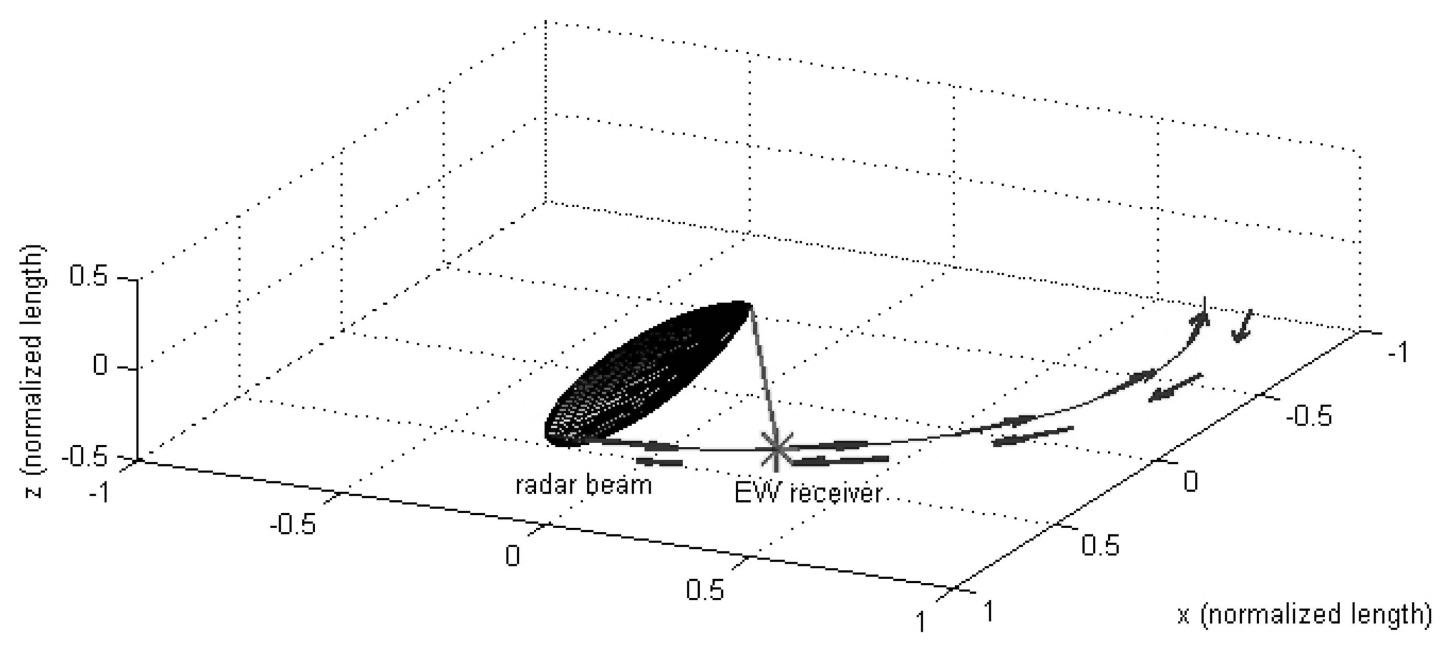

$y$ (nomalized length)

(a)

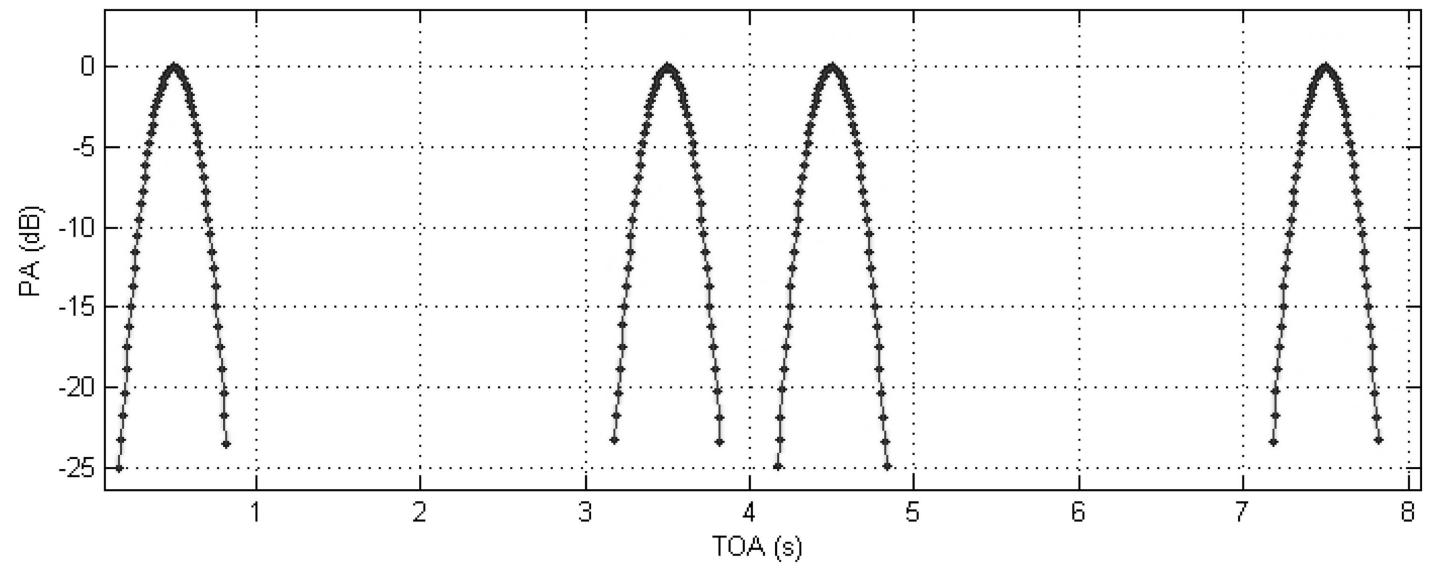

(b)

Fig. 2. Sector scan. (a) Main beam positions. (b) PA versus ToA graph.

measured from the normal to the antenna [18]. In addition to the changing shape of the main beam, the sidelobes also change in appearance and position. Consequently, the antenna scan pattern received at the EW receiver keeps changing, complicating the ASP estimation and AST recognition problem. Instead of ASP and AST, one can use lobe duration (illumination time) as a distinctive feature of phased-array radars. However, one should consider that the lobe duration may also vary (probably small variations) because of the beam broadening nature of phased arrays when looking off the boresight of the array.

\section{ANTENNA SCAN PATTERN SIMULATOR}

Because of the scarcity of recorded EW receiver data, an antenna scan pattern simulator (ASPS) with a graphical user interface (GUI) is developed using MATLAB [26]. An example screen of the ASPS is illustrated in Fig. 6 where the different parts of the GUI can be seen on the left, and a sample PA versus ToA plot is shown on the right.
The transmitter antenna of the radar is modeled as a linear antenna array, with optional weightings (uniform, triangle, Hamming, Taylor, Poussin, and Blackman) that determine the sidelobe level of the antenna pattern. The simulator calculates the number of elements needed to achieve the desired azimuth and elevation beamwidths for a particular model and synthesizes a pattern for them.

The ASTs described in Section III are simulated by the ASPS. According to the AST selected by the user, different edit boxes are enabled with corresponding parameter names. The user inputs the desired parameters for the particular scan type. Period is the common parameter for all of the ASTs. Sector scan requires the start-finish positions in the azimuth and the elevation position of the beam. Raster scan acquires the start-finish positions in azimuth and elevation with the number of bars in the elevation. Conical scan type needs the target azimuth and elevation position and squint angle of the radar. All of the above parameters are used to calculate the beam position at a specific point in time corresponding to 


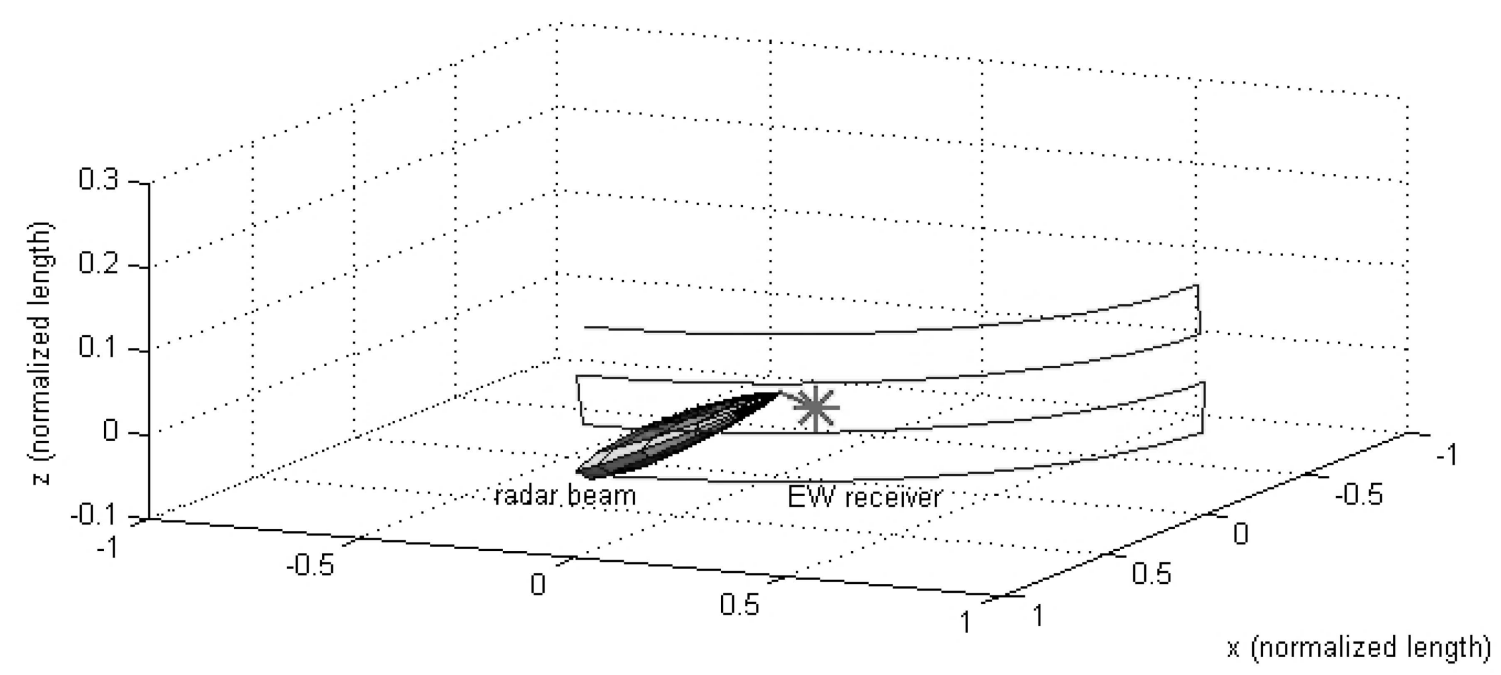

$y$ (normalized length)

(a)

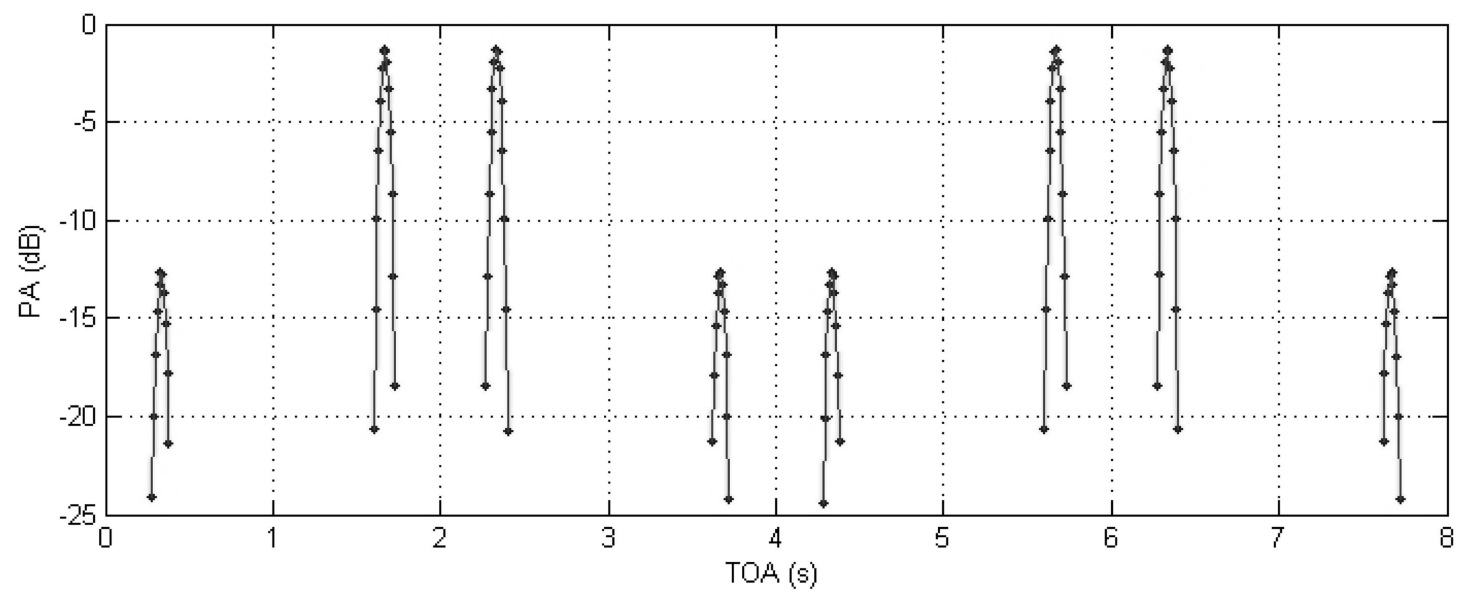

(b)

Fig. 3. Raster scan. (a) Main beam positions. (b) PA versus ToA graph.

the ToA of the pulse. The user can input the ToAs of the radar pulses that determine the sampling points of the antenna scan pattern.

As the radar antenna rotates, the gain observed by the EW receiver (that is the $G_{t}(\theta(t), \phi(t))$ factor in the received power in (1)) changes with time. The simulator calculates the beam positions for each pulse according to the selected AST and ASP. The properties and the position of the EW receiver with respect to the radar are given as input. The simulator then calculates the gain observed at the EW receiver using the receiver's azimuthal and elevational position with respect to the radar. The radar is assumed to be at the origin of the coordinate system. Then, the received power at the EW receiver is calculated for each pulse of the radar using its ToA and the beam's position using (1). PAs are normalized so that the pulse with the maximum power corresponds to $0 \mathrm{~dB}$.

The EW receiver antenna is modeled as isotropic (i.e., constant $G_{r}$ ) and the propagation loss is assumed to be constant. It is assumed that the EW receiver remains stationary (or moves a negligible amount compared with the radar) so that the range $R$ is approximately constant throughout the simulation time. The noise level in the EW receiver is simulated by the addition of zero-mean Gaussian noise with the desired power to the signal. The sensitivity level of the receiver can be adjusted as well. Signals with amplitudes below the sensitivity level of the receiver are not detected.

Unmodulated pulses as well as pulses with both intrapulse and interpulse modulation have been implemented. The resulting PA versus ToA graphs of the simulated scenarios can be plotted and explored. An example is provided in Fig. 6.

The simulator also illustrates how the beam scans the volume in time. It divides the whole simulation time into 10 intervals and illustrates the beam position at these time points and also plots the position of the EW receiver to see the effects of the rotation of the beam. 


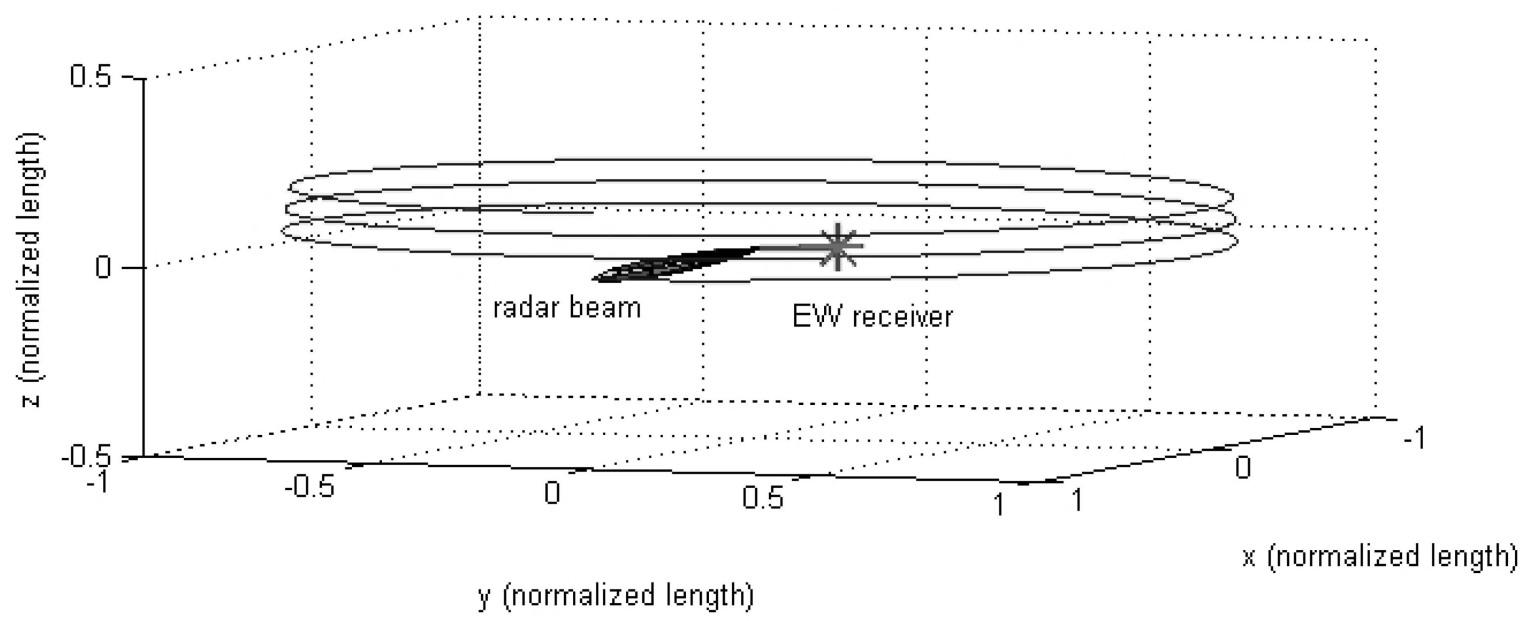

(a)

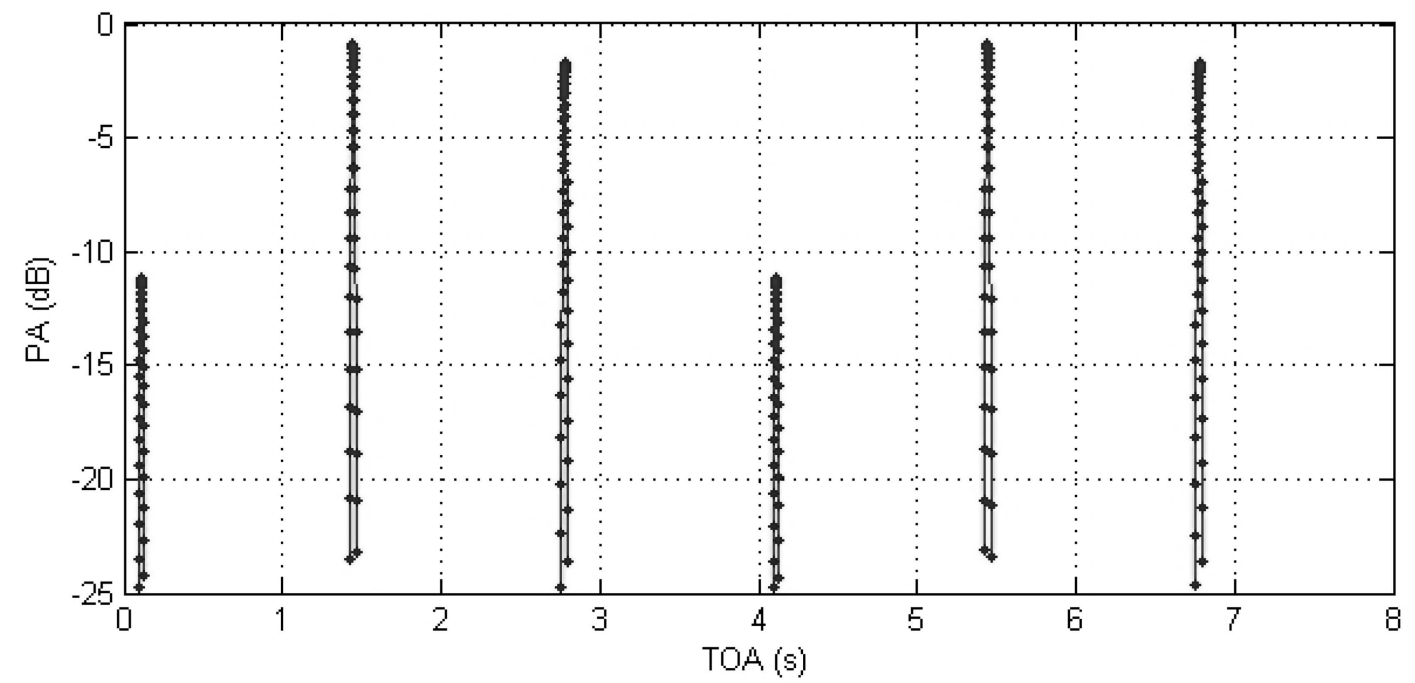

(b)

Fig. 4. Helical scan. (a) Main beam positions. (b) PA versus ToA graph.

\section{AST RECOGNITION ALGORITHM}

The AST recognition problem can be summarized as estimating the relative angular position of the EW receiver with respect to the radar main beam as time passes, and classifying the radar AST into one of the most frequently encountered scan patterns. Although it should be possible to infer the AST based on the power received from the radar, the problem is complicated in multiple ways: the location of the radar antenna and its radiation pattern are completely unknown to the EW receiver, and the parameters of the ASTs vary vastly (ASP, number of bars in raster, sector width, etc.). Trying to check all possible received power outcomes and defining a metric (e.g., Euclidean distance) to measure the similarities would exhaust any computation power feasible. This deficiency of vital information has led the study to suboptimal solutions, in which several characteristic features of the radar signal are used. An overview of the proposed algorithm is given in Fig. 7.

\section{A. The Input Signal}

The input PA versus the ToA data is either the output of the ASPS or is real data acquired by the EW receiver. Figure 8(a) shows an example PA versus a ToA signal from a raster scan where three periods are shown. Since the output from these sources are in $\mathrm{dBW}$ (decibel relative to Watt), the signal is first transformed from dBW to Volt (V) scale using $10^{\text {(power/20). }}$

Although uniform sampling is employed in many applications, in some cases, nonuniformly sampled signals can be encountered. Estimating the period of a uniformly sampled signal is much simpler and usually independent from the method used. If the signal is nonuniformly sampled, it is better to resample it uniformly. For example, signals with stagger-type PRI result in nonuniform sampling. If this is the case, the signal is resampled with the highest PRI in the stagger levels. During the resampling process, if the signal value is not available at a particular instant, interpolation is performed using the two nearest 


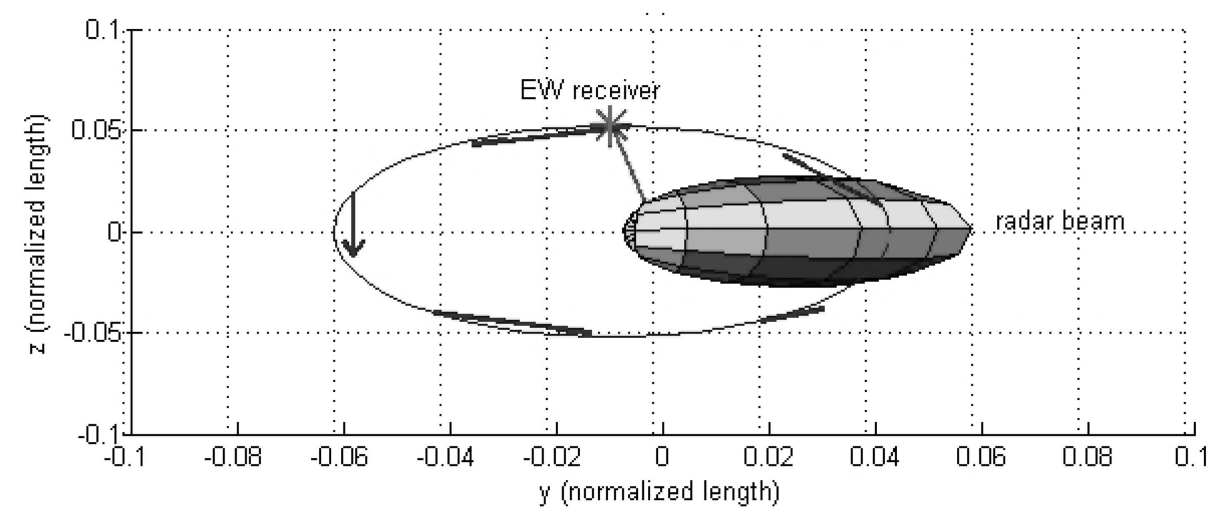

(a)

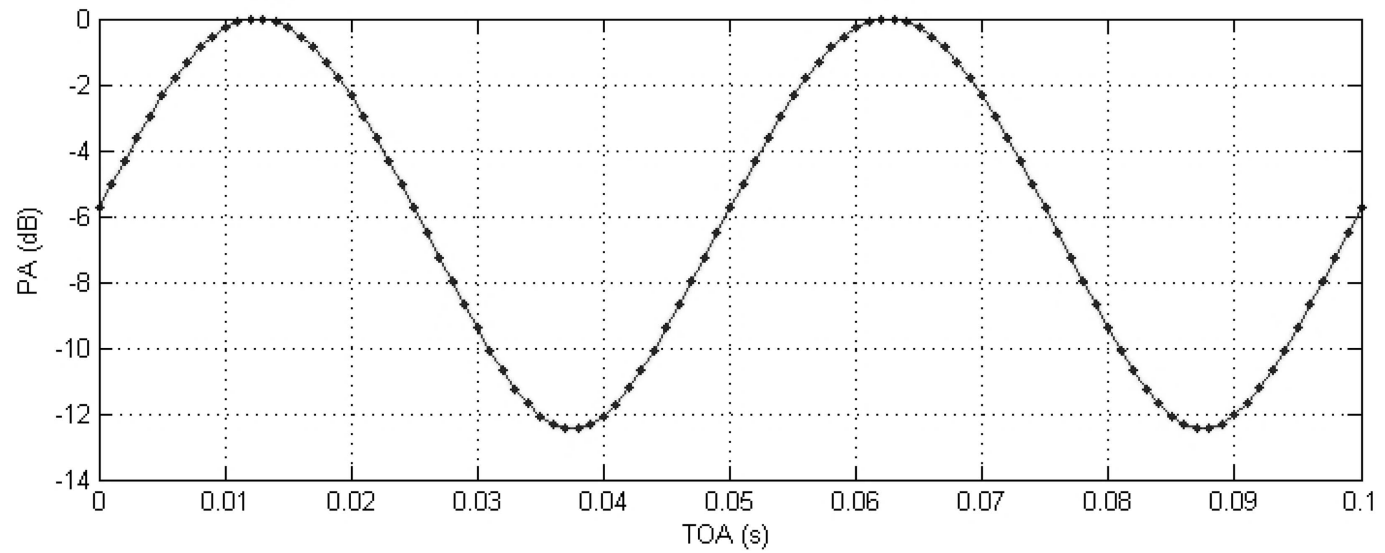

(b)

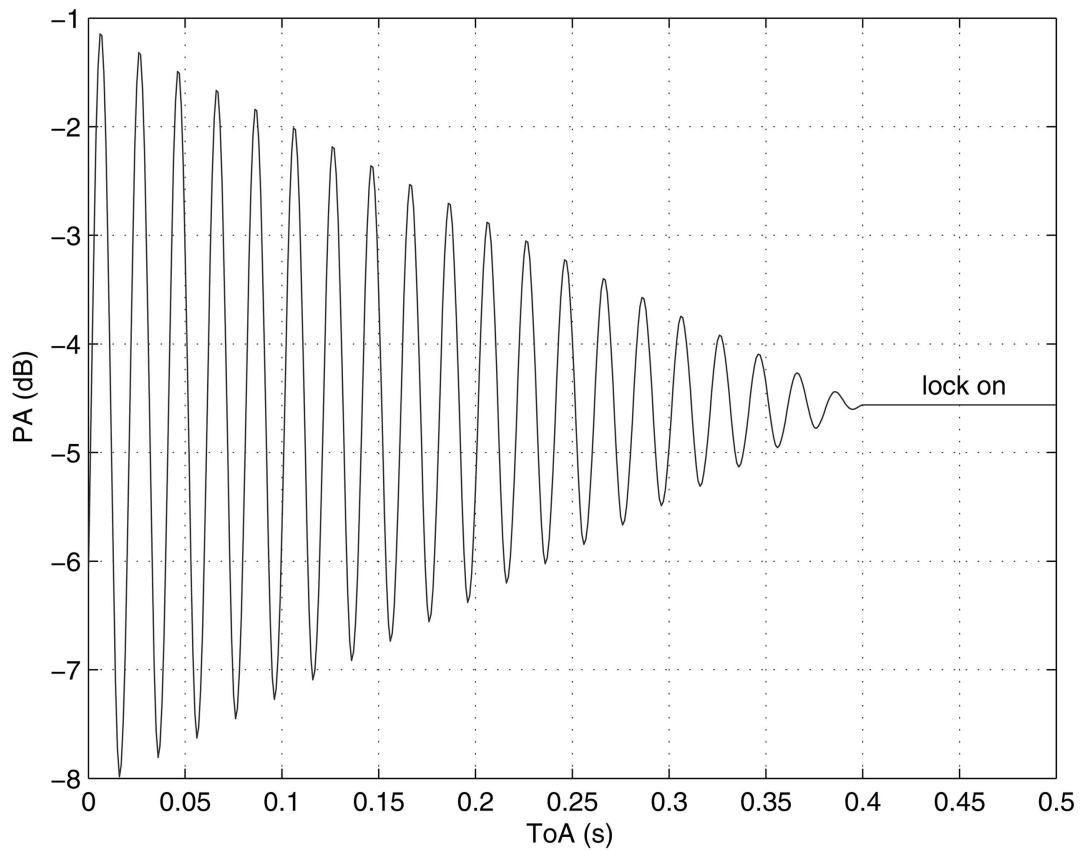

(c)

Fig. 5. Conical scan. (a) Main beam positions. (b) PA versus ToA graph. (c) Lock-on process.

points. Other interpolation techniques can also be used in resampling (see Section V-B). In some cases, especially when the antenna is looking away from the EW receiver, the receiver does not detect any pulses. These points are filled with zeros to make the succeeding computations easier. As a result of these steps, the example sequence in Fig. 8(a) is transformed to the sequence $x[n]=x\left(n T_{s}\right)$ in Fig. 8(b), where $n=0,1, \ldots, N-1$ and $T_{s}$ is the sampling period. 


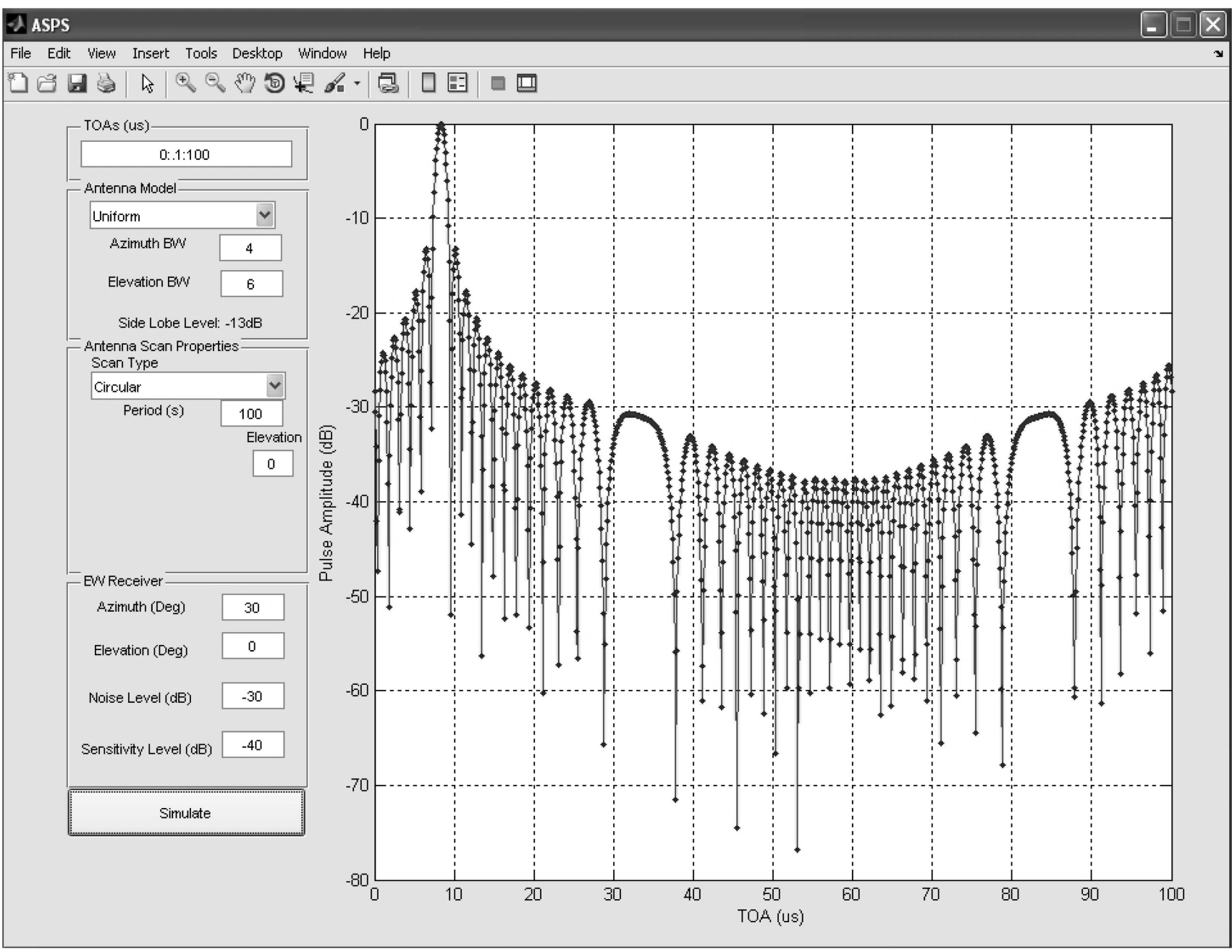

Fig. 6. ASPS GUI: Different parts of GUI can be seen on left, and sample PA versus ToA plot is shown on right.

The input signals are synthesized by using the ASPS described in the previous section. Parameters of the synthesized signals (e.g., the PRI, ASP, azimuth and elevation beamwidths of the radar antenna, number of bars in raster, sector width) are selected realistically over a wide range, taking real radar systems as examples. We used a classified database for this purpose.

\section{B. Period Estimation}

A continuous-time signal $x(t)$ is called periodic if $x(t)=x(t+T) \forall t$ and some period value $T$. The smallest value of $T$ that satisfies this equality is called the fundamental period. Period estimation has attracted considerable interest over the years, especially in the speech processing area [27-31]. The problem is sometimes called (fundamental) tone estimation or pitch estimation. There are solutions to the problem in the time and the frequency domains.

Frequency-domain methods estimate the period by detecting the peaks of the frequency spectrum. This approach possesses some problems when the signal is not a sinusoid, but has a wide bandwidth. In this case, the peaks may be illusive when estimating the fundamental frequency.

Time-domain methods are particularly useful for period estimation of nonsinusoidal signals. These methods define some kind of similarity metric and try to maximize the similarity with the lagged versions of the signal using this metric. For example, the average magnitude difference or the autocorrelation between the signal and its lagged versions can be used as similarity metrics. The lag value where the similarity is maximized corresponds to the period estimate of the signal. The latter approach provides the best performance when the noise for each of the two signals is white Gaussian noise (which usually is the case).

The backbone of our algorithm is the ASP estimation, which affects the overall performance of AST classification significantly. An ASP has a very large range whose true value is usually acquired from intelligence agencies. The ASP estimation method should be chosen according to the properties of the signal. In this study period estimation is performed by using the normalized autocorrelation coefficients of 


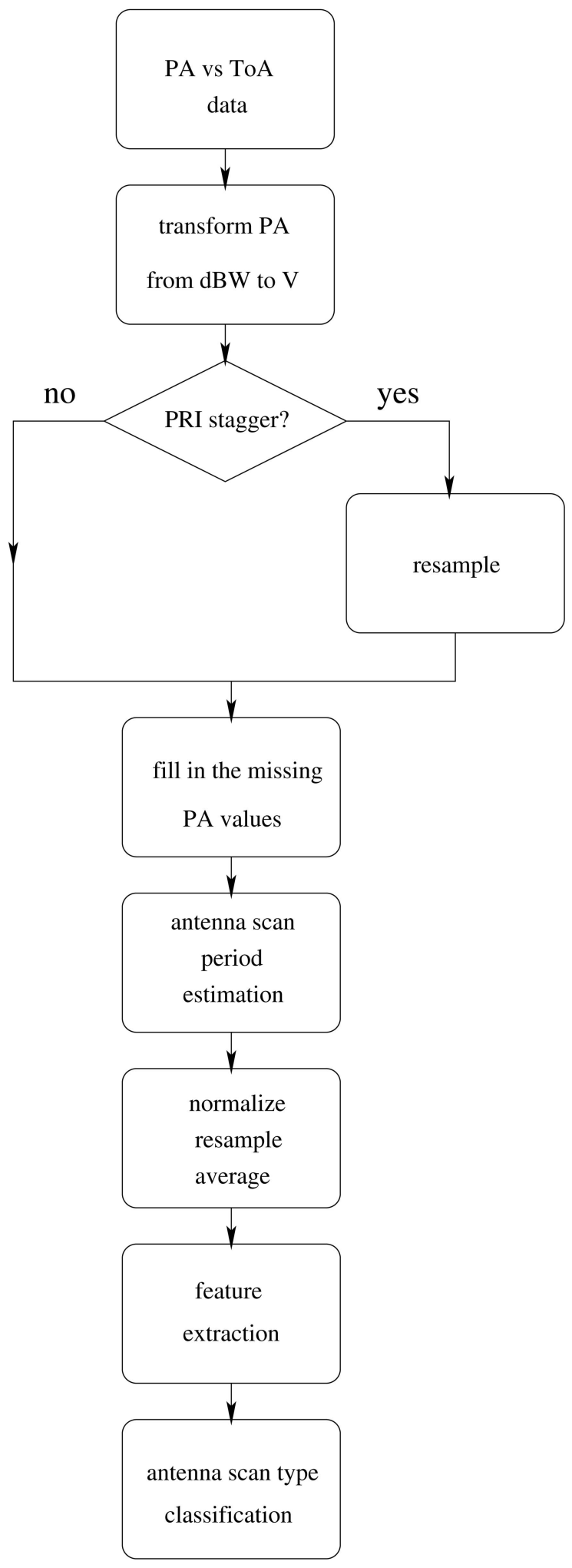

Fig. 7. Overview of AST recognition algorithm.

the sequence $x[n]$, calculated as follows:

$$
\begin{array}{r}
r_{x x}[l]=\frac{\sum_{n=0}^{W-1} x[n] x[n+l]}{\sqrt{\sum_{n=0}^{W-1} x^{2}[n]} \sqrt{\sum_{n=0}^{W-1} x^{2}[n+l]}}, \\
l=0,1, \ldots, N-W .
\end{array}
$$

Here, $l$ is the lag variable and $W$ is the window size that should be chosen to compromise between computational complexity and accuracy. The sequence $R_{x x}[l]$ is thresholded to find the maximum correlation coefficient and the corresponding lag value is assigned as the period. Figure 9 illustrates $r_{x x}[l]$ for the example signal in Fig. 8, which has a total length of $N=7,500$ samples. Using a threshold level of 0.98 and $W=N / 3$, the period is estimated to be 2,500 lags.

\section{Preprocessing (Normalization, Resampling, and Averaging)}

The preprocessing stage before feature extraction involves normalizing, resampling, and averaging the sequence. This is done to reduce the effect of noise and transform the signal into standard dimensions, eventually improving the quality of the features.

The signal amplitude is first normalized by its maximum value so that it varies between zero and one and so that the feature selection process is not affected by the amplitude range of the signal:

$$
x_{n}[n]=\frac{x[n]}{\max (x[n])}, \quad n=0,1, \ldots, N-1 .
$$

The normalization process helps in removing the effects of propagation loss, since this results in a decay in PA proportionate to the range $R$ between the radar and the EW receiver.

The input signal is typically from a set where the sampling rates and the number of samples are widely varying, as is the case in our application. To extract informative and discriminating features from this type of signal, it is better to have the same number of samples acquired at similar sampling rates. This improves the quality of the features and reduces their dependence on the length of the signal and the sampling rate.

Resampling (or sample rate conversion) is the process of converting the sampling rate of a (usually digital) signal from one value to another. Modern systems mostly use digital signal processing techniques since this approach introduces less noise and distortion compared with first converting the signal to analog and then resampling at a new rate. The signal can be treated as a time series and may be resampled at a new sampling rate using more samples (which is called interpolation or upsampling) or fewer samples (which is called decimation or downsampling) [32].

Resampling is performed here to be able to uniformly interpret various radar signals with different ASPs and sampling intervals. The resampling process transforms all recorded signals from various radar with different PRIs, ASTs, and ASPs with $T / T_{s}$ elements per period into a standard sequence of $M$ elements per period. The sampling interval after the resampling process becomes $T_{o} \triangleq T / M$ where $T$ is the ASP of the radar. The resampling process used here can be considered as a decimation operation that 


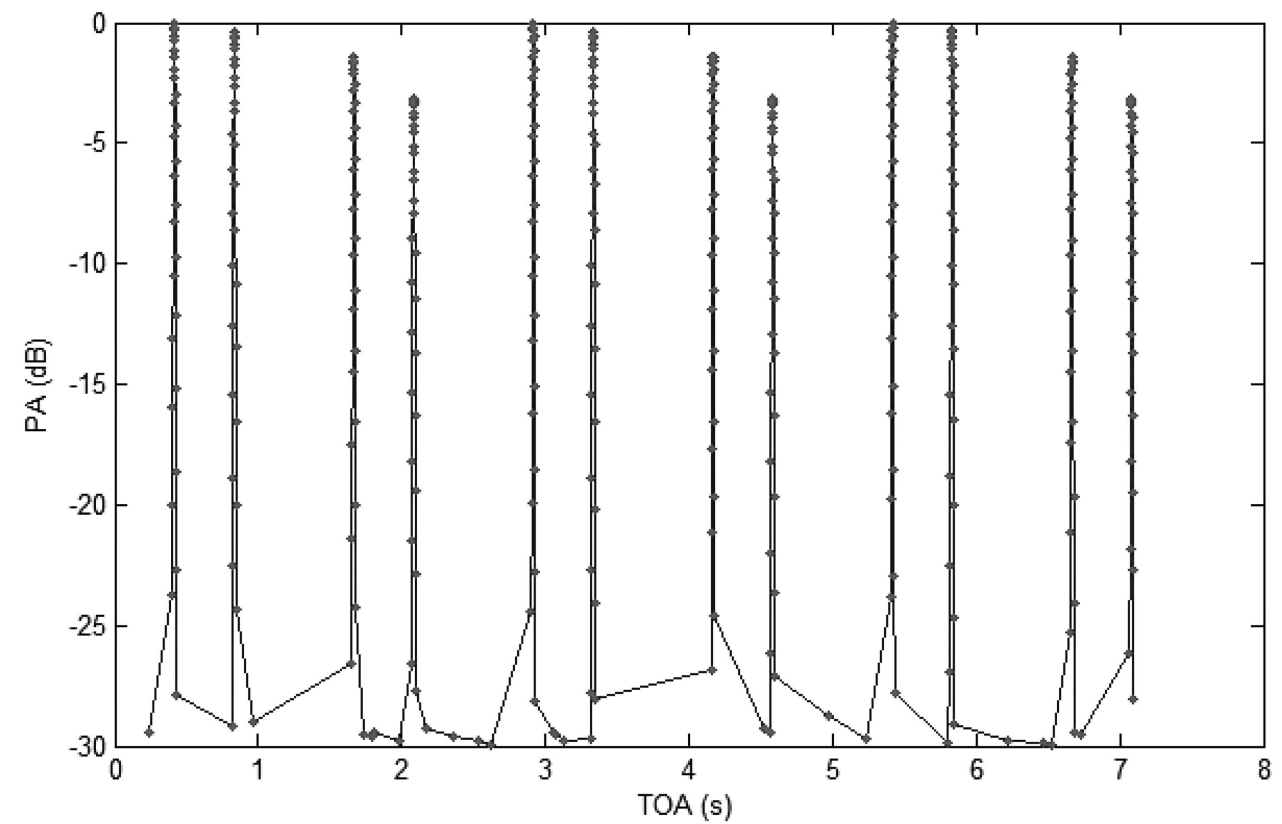

(a)

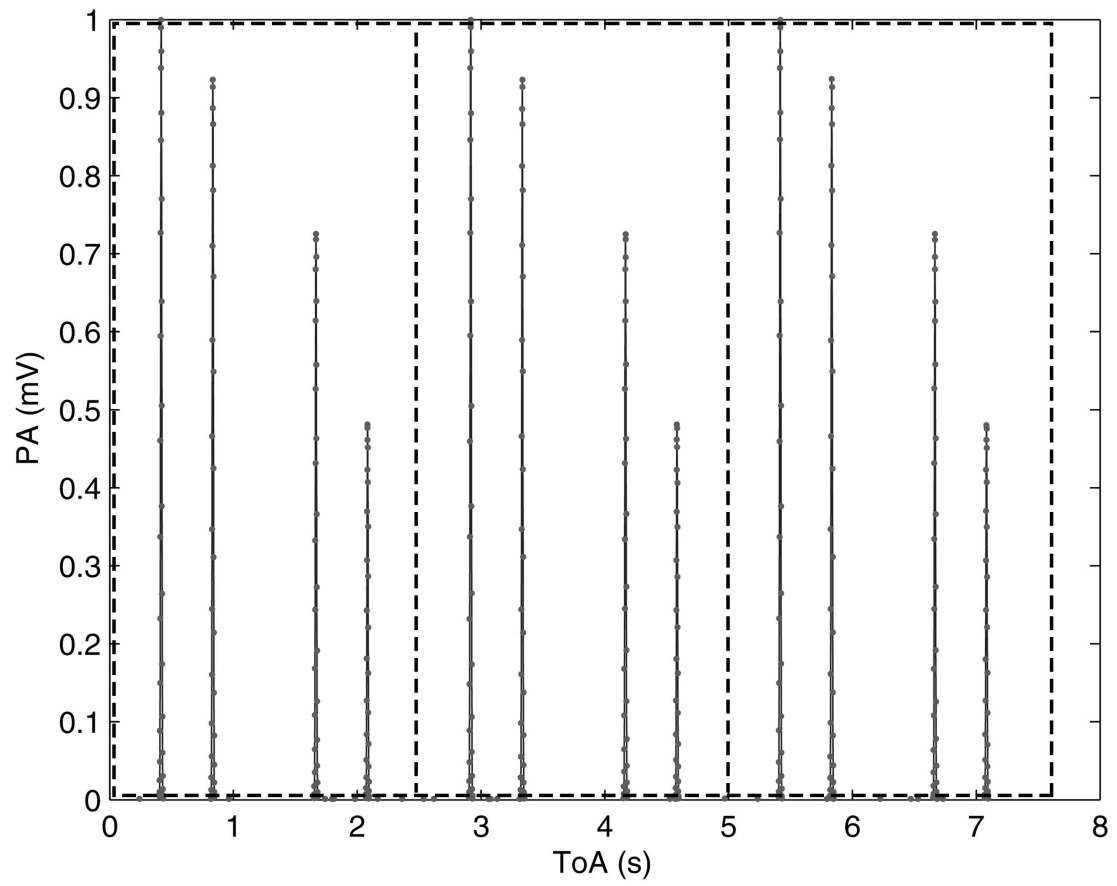

(b)

Fig. 8. (a) Example signal from raster scan, where solid dots indicate PAs. (b) Transformed signal ready for period estimation, where three periods separated by vertical dashed lines are shown.

reduces the amount of data because radars usually use a large number of pulses per scan period to closely track the changes in the environment.

We assume that several periods of a particular radar scan pattern are available, the total number of samples being $N$ and the sampling interval being $T_{s}$. After resampling, the total number of samples per period of any type of scan will become $M$. A suitable value of $M$ is determined beforehand by analyzing the patterns and the sampling interval is changed from $T_{s}$ to $T_{o}$. While doing this, the values of any needed new points can be calculated by interpolation. Different interpolation techniques such as nearest point (nearest neighbor), linear, polynomial, spline, Gaussian, or sinc interpolation can be used. In this study we chose to use nearest point interpolation for its simplicity:

$$
\begin{aligned}
x_{r}[m]= & x_{n}\left[\operatorname{round}\left(\frac{m T_{o}}{T_{s}}\right)\right], \\
& m=0,1, \ldots,\left\lfloor\frac{(N-1) T_{s}}{T_{o}}\right\rfloor
\end{aligned}
$$




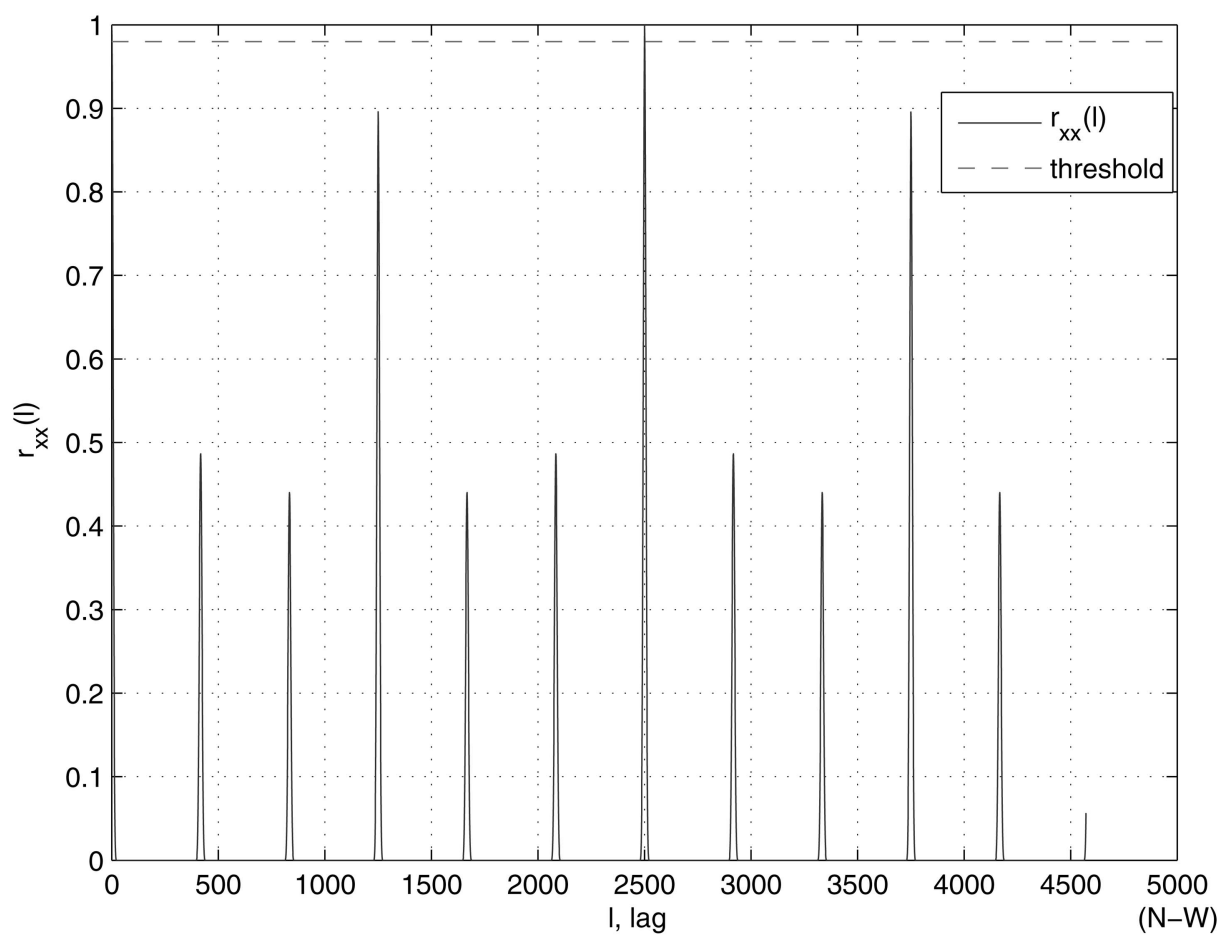

Fig. 9. Normalized autocorrelation coefficients of example sequence.

where $x_{r}[m]$ denotes the resampled sequence and $\lfloor$. indicates truncation. The nearest point interpolation rounds off noninteger $m T_{o} / T_{s}$ values to the nearest integer so that the resampled sequence at a specific point $m$ takes the value of its nearest neighbor in the normalized sequence. The values of the other neighboring points are not considered. This type of interpolation has the advantage of being simple and fast. Because a high sampling rate is available before the resampling process, the nearest point interpolation technique results in negligible distortion in the signal.

It is assumed that the input signal has at least two cycles of the antenna scan. This is important because the period estimation phase needs at least a few cycles to reduce the effect of noise and to accurately estimate the signal period. If at least $K$ complete periods are available for period estimation so that $N T_{s} \geq K T$, these can be coherently averaged as

$$
\bar{x}[m]=\frac{1}{K} \sum_{k=0}^{K-1} x_{r}[m+k M], \quad m=0,1, \ldots, M-1 .
$$

The example sequence after normalization, resampling, and averaging over three periods is depicted in Fig. 10 where an $M$ value of 2,000 is used. From this point on in the text, the word "signal" refers to this averaged sequence.

\section{Feature Extraction}

Input to the feature extraction part is the preprocessed sequence with $M$ elements and sampling interval $T_{o}$. A total of 100 data sequences is generated by the ASPS, 20 from each of the following ASTs in the given order: circular, sector, raster, helical, and conical. While generating the simulated training data, we have varied a number of different parameters so that the training samples resemble the real data from different realistic scenarios as much as possible. For the four ASTs other than the conical, the ASP is uniformly varied between 1-10 s (ASR: $0.1-1 \mathrm{~Hz}$ ). For the conical scan (whose scan rate is much faster compared with the other scans), the ASP is uniformly varied between $0.01-0.2 \mathrm{~s}$ (ASR: 5-100 Hz) in generating the 20 training data samples.

The azimuth and elevation sector widths, EW position with respect to the radar, and the squint angle related to the radar-EW receiver engagement have also been varied. For the circular antenna scan pattern, the azimuth angle has a uniform random distribution in $\left[0,360^{\circ}\right]$. For the sector scan, different sector widths in $\left[0,180^{\circ}\right]$ are used and the position of the EW receiver within the sector is randomly selected with uniform distribution. For the raster scan, the azimuth is varied in $\left[0,180^{\circ}\right]$, whereas for the helical scan, the corresponding interval is $\left[0,360^{\circ}\right]$. In the latter two ASTs, the elevation is varied in $\left[0,10^{\circ}\right]$ and again, the position of the EW receiver is randomly selected. For the conical scan, random locations around the position tracked by the radar are used for the EW receiver.

Features such as the minimum and the maximum values, mean, standard deviation, skewness, kurtosis, or higher order moments are commonly employed to describe the probability density function of the signal. Coefficients of various transforms such as FFT, 


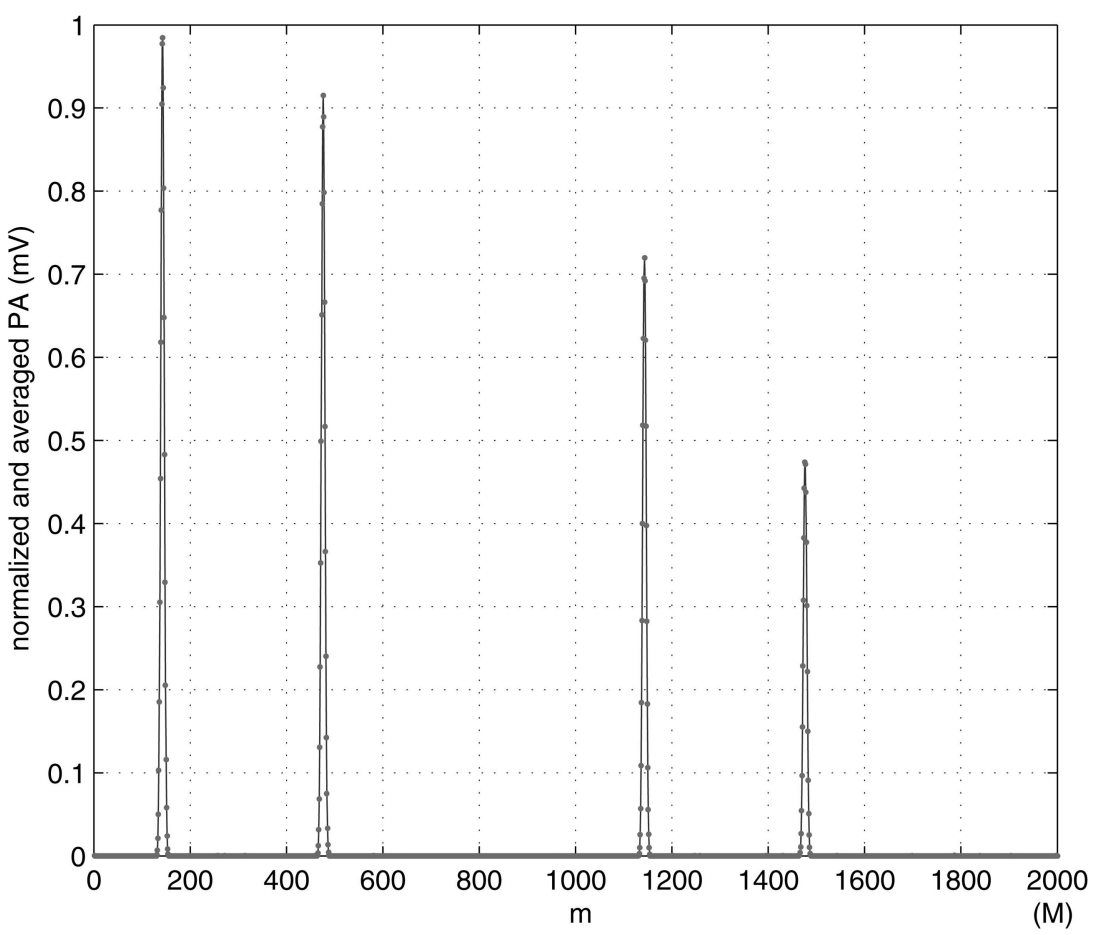

Fig. 10. Sequence $\bar{x}[m]$ where main beam is leftmost peak.

DWT, and discrete cosine transform (DCT) are also widely used. The most informative and discriminative features depend on the physical phenomena that affect the classes. We have attempted to use a number of different features such as the mean, standard deviation, and skewness, but the best results were obtained with the four features described below.

Kurtosis is the normalized fourth-moment of a random variable $Z$ and is defined as $E\{Z-\mu\}^{4} / \sigma^{4}$, where $E\{\cdot\}$ denotes the expected value, $\mu$ is the mean and $\sigma$ is the standard deviation of the random variable. Kurtosis is a statistical measure of how peaked or how flat the distribution of the random variable is. It can also be considered as a measure of how heavy the tails of the distribution are relative to the Gaussian distribution, which has a kurtosis value of three. Concentrated distributions such as uniform distribution have kurtosis values less than three. If the distribution has heavier tails and is more outlier prone, its kurtosis value exceeds three.

In track modes radar tries to illuminate the threat as much as possible so that it can update the threat's coordinates accurately in the event of an attack. To perform this task, it must steer the beam such that it is always focused on the platform. This in turn means that the EW receiver will receive the pulses of the radar with slight differences in amplitude (i.e., a narrow amplitude distribution), depending on the track scan type of the radar system. The amplitude distribution is approximately uniform. A conical scan signal, which is a uniformly sampled sinusoid, has a kurtosis value of around 1.5. By observing the signal's kurtosis value, the system will infer its mode and AST. The kurtosis value will be small for the conical scan type and large otherwise, indicating that kurtosis of the sequence can be used as a discriminating feature for the conical scan. This is depicted in Fig. 11(a). Therefore, we select the kurtosis of $\bar{x}[m]$ as the first feature.

It can be observed by analyzing different AST patterns that the number of main beams, their amplitude variation, and separation in time are the key parameters for differentiating the basic ASTs. First, the algorithm detects the main beam in the signal by finding its maximum value. Using the two index points on each side of the maximum point where the PA drops to 0.01 , the main beam sequence $y[\mathrm{~m}]$ of length $V$ is extracted (leftmost peak in Fig. 10).

After finding the main beam with the maximum amplitude, the algorithm detects the other main beams in the sequence using the normalized cross correlation between the sequence $\bar{x}[m]$ and the detected main beam $y[m]$ :

$$
\begin{array}{r}
r_{\bar{x} y}[l]=\frac{\sum_{m=0}^{V-1} \bar{x}[m+l] y[m]}{\sqrt{\sum_{m=0}^{V-1} \bar{x}^{2}[m+l]} \sqrt{\sum_{m=0}^{V-1} y^{2}[m]}}, \\
l=0,1, \ldots, N-V
\end{array}
$$

where $l$ is again the lag variable and $V$ is the length of the main beam vector $y[m]$. Setting a threshold and finding the peaks in the cross-correlation values identifies all possible main beams.

One would expect the cross-correlation value to be large since the patterns of different antennas are very similar near their boresights. For scan types where 


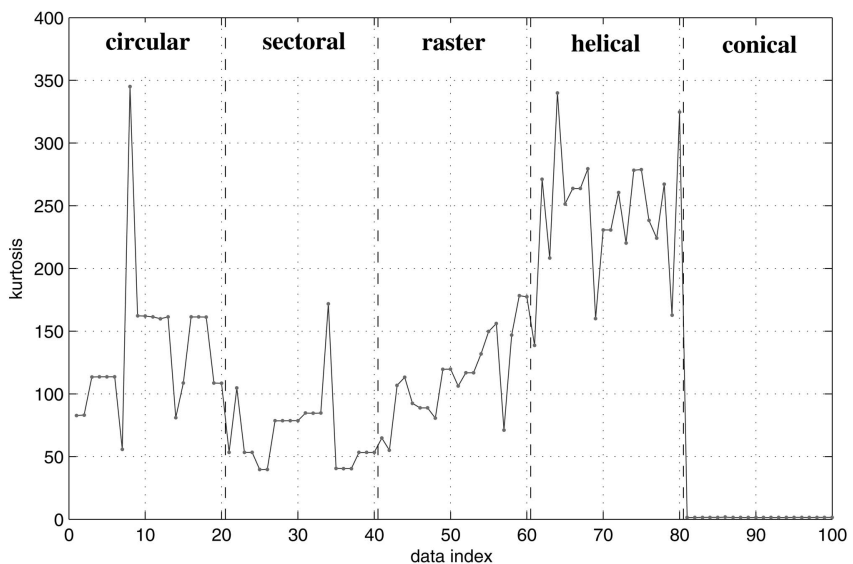

(a)

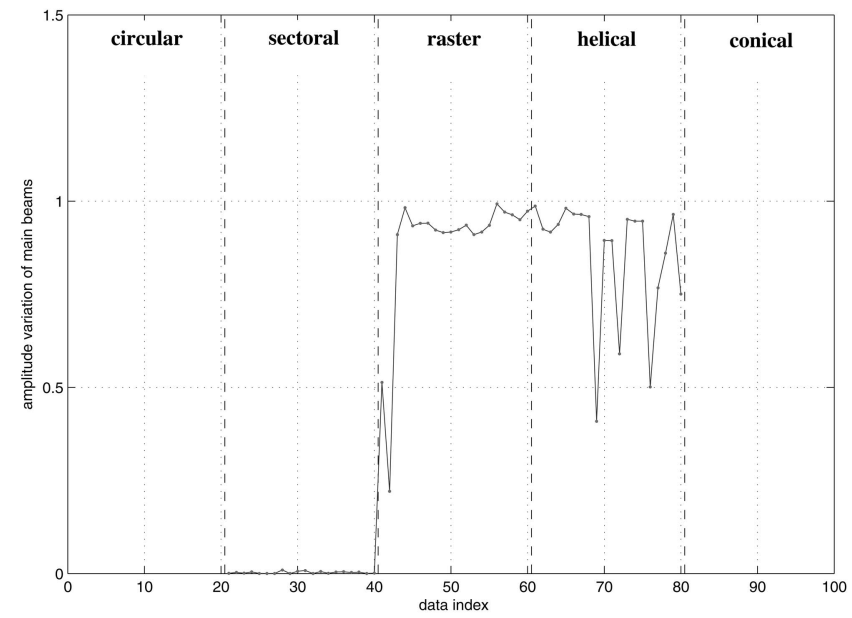

(c)

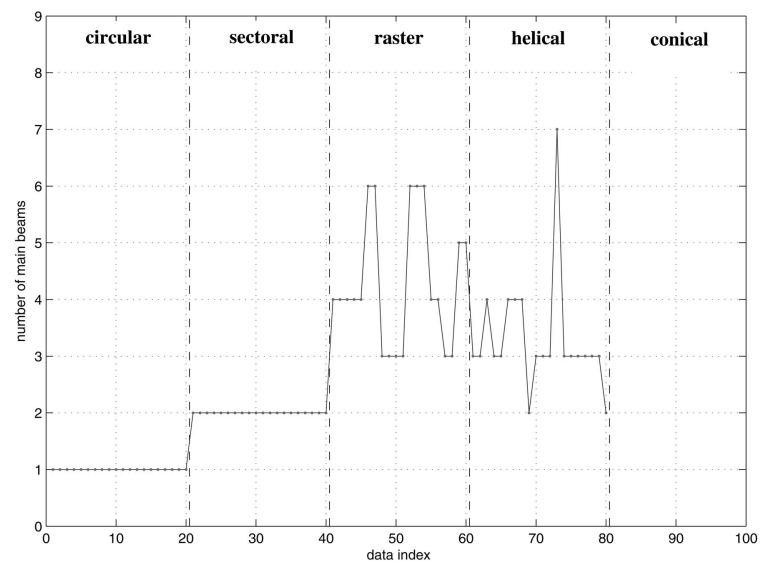

(b)

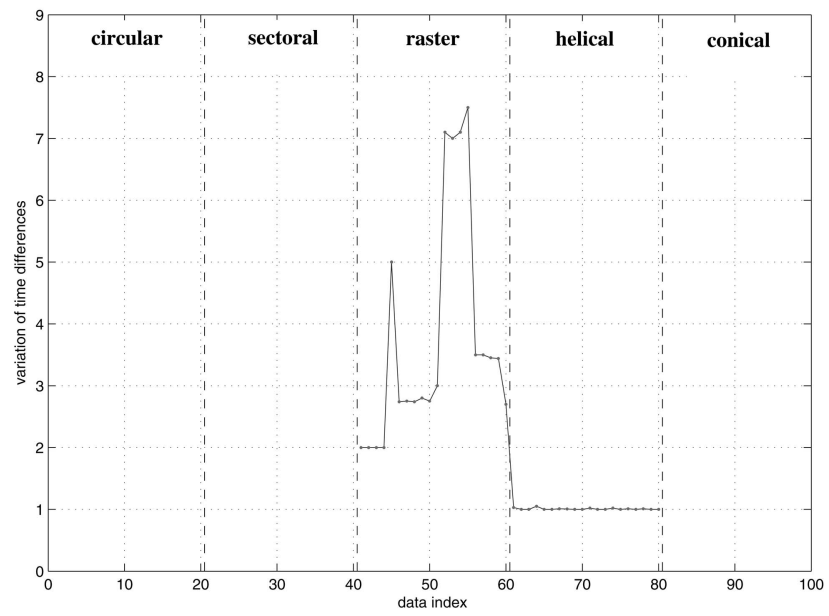

(d)

Fig. 11. (a) Kurtosis values. (b) Number of main beams. (c) Amplitude variation of main beams. (d) Time differences between main beams in signal.

no elevational action is involved (circular and sector), the main beams will be exactly the same, ignoring the effect of noise. The threshold can be tuned according to the signal to be able to handle possible variations in the azimuth pattern caused by the changing elevation angle. In the example signal in Fig. 10, a threshold value of 0.95 is used and altogether four main beams are detected, corresponding to the visible peaks of the signal.

The number of main beams is an important parameter that can differentiate some ASTs from the others. In particular, the circular scan has only one and the sectoral scan has two main beams in one period. Figure 11(b) displays this feature for all the scan data and indicates that this feature can be very valuable for AST classification. Circular and sector scan types are highly differentiable by this parameter. It can also be observed that no main beam is found in the conical signal since pulse variation is not observed. Therefore, we choose the second feature as the number of main beams in the signal.

Amplitude variation of the main beams can also be a very useful feature to differentiate between the ASTs that involve only one plane (azimuth) and the ones that scan both planes (azimuth and elevation). Therefore, the third feature is selected as the amplitude variation of the main beams calculated as the difference between the minimum and maximum main beam amplitudes: max(main beam PAs) min(main beam PAs). Figure 11(c) shows the distribution of this feature for all data sequences. Unlike the other scan types, the value of this feature fluctuates around zero for the sector scan. Therefore, this scan type can be easily differentiated from the others using this feature. One can see that the variation could not be calculated for the circular scan type since this type of scan produces only a single main beam. Similarly, since the conical scan does not have any distinct main beam, variation of the main beams is not calculated for this AST either.

Time difference between the main beams is another distinctive parameter between classes. Circular and helical scan types continuously revolve $360^{\circ}$ without going back and forth as sector and raster scans do. In this case, we expect a constant time difference between the main beams. However, in the 
raster scan, the time difference between the main beams usually varies because of the nature of the scan. The only case where the time difference will be the same is when the EW receiver is in the middle of the scanned sector, and this is unlikely. Therefore, the ratio of the maximum of the time differences to the minimum is calculated and used as the fourth feature. Note that this feature is only calculable for helical and raster scans since there should be at least three main beams to calculate the variation of time differences between the main beams (Fig. 11(d)). For features that cannot be calculated for some of the ASTs, a value of 10,000 is used as an indicator to the classification stage.

\section{E. Classification}

We associate a class $c_{i}$ with each AST ( $i=$ $\left.1, \ldots, N_{c}\right)$. An unknown AST is assigned to class $c_{i}$ if its feature vector $\mathbf{z}=\left[z_{1}, \ldots, z_{N_{F}}\right]^{T}$ falls in the region $\Omega_{i}$. A rule that partitions the decision space into regions $\Omega_{i}, i=1, \ldots, N_{c}$ is called a decision rule. Each one of these regions corresponds to a different AST. Boundaries between these regions are called decision surfaces.

The training set, which consists of a total of $N_{p}$ sample feature vectors, is used to develop the decision rule or to train the classifier. The test set is then used to evaluate the performance of the classifier.

The four selected features are used in the classification process with the naive Bayes (NB), decision tree (DT), ANN, and support vector machine (SVM) classifiers [33]:

1) Naive Bayes Classifier: In classification problems, when there are more than a few features and classes, usually a large number of observations is needed to estimate the probabilities of a pattern belonging to different classes. The NB classifier is a simple probabilistic classifier that gets around this problem by not requiring a large number of observations for each possible combination of the features [34]. It is one of the most efficient and effective inductive learning algorithms for machine learning and data mining that is particularly suitable when the dimensionality of the features is high. Parameters of the probabilistic model are calculated under the assumption that the features are independent of each other. In other words, an NB classifier assumes that the presence (or absence) of a particular feature of a class is unrelated to the presence (or absence) of any other feature. The features independently contribute to the probability of a pattern belonging to different classes. Although this assumption is rarely true in real-world applications, it is made to simplify the computations and in this sense considered to be naive.

NB classifiers can be trained very efficiently with fewer observations in a supervised learning setting.
Despite their naive design, simplicity, and apparently oversimplified assumptions, these classifiers work surprisingly well in many complex real-world situations and can often outperform more sophisticated classification methods.

Given the classes $c_{1}, c_{2}, \ldots, c_{N_{c}}$, let $p\left(c_{i}\right)$ be the a priori probability of an AST belonging to class $c_{i}$. To classify an AST with feature vector $\mathbf{z}$, a posteriori probabilities $p\left(c_{i} \mid \mathbf{z}\right)$ are compared and the scan pattern is classified into class $c_{j}$ if $p\left(c_{j} \mid \mathbf{z}\right)>p\left(c_{i} \mid \mathbf{z}\right)$ $\forall i \neq j$. This is known as Bayes minimum error rule. However, since these a posteriori probabilities are rarely known, they need to be estimated. A more convenient formulation of this rule can be obtained by using Bayes' theorem: $p\left(c_{i} \mid \mathbf{z}\right)=p\left(\mathbf{z} \mid c_{i}\right) p\left(c_{i}\right) / p(\mathbf{z})$, where $p(\mathbf{z})=\sum_{i=1}^{N_{c}} p\left(\mathbf{z} \mid c_{i}\right) p\left(c_{i}\right)$ is the total probability. This results in $p\left(\mathbf{z} \mid c_{j}\right) p\left(c_{j}\right)>p\left(\mathbf{z} \mid c_{i}\right) p\left(c_{i}\right) \forall i \neq j$ $\Rightarrow \mathbf{z} \in \Omega_{j}$ where $p\left(\mathbf{z} \mid c_{i}\right)$ are the class-conditional probability density functions that are also unknown and need to be estimated in their turn based on the training set. Probabilistic models for $p\left(\mathbf{z} \mid c_{i}\right)$ are developed first, using the available training data for each class. As mentioned above, a major advantage of the NB classifier is that it requires only a small amount of training data to estimate the parameters (means and variances of the features) necessary for classification. Since features are assumed to be independent, it is not necessary to estimate the entire covariance matrix; only the variances of the features for each class need to be determined. NB can be modeled in several different ways including normal, lognormal, gamma, and Poisson density functions. In this study, the probability density functions are modeled as normal distributions whose parameters (mean and variance) are calculated by maximum likelihood estimation. Then, the posterior probabilities are calculated according to the probabilistic models of each class by applying the Bayes' theorem given above. The class of the signal is chosen as the one with the highest posterior probability according to Bayes minimum error rule. Thus, the decision rule for classification is merely picking the hypothesis that is the most probable. This decision rule can be generalized as $q_{j}(\mathbf{z})>q_{i}(\mathbf{z}) \forall i \neq j \Rightarrow \mathbf{z} \in \Omega_{j}$ where the function $q_{i}$ is called a discriminant function.

2) Decision-Tree Classifier: DT classifiers are one of the most intuitive and natural classifiers and are fast, comprehensible, and easy to visualize [33]. A DT can be considered as a sequential procedure to classify given input patterns. It follows predefined rules or test conditions at each node of the tree and makes binary decisions based on these conditions. Rules correspond to conditions such as "is feature $z_{i} \leq \tau_{i}$ ?," where $\tau$ is the threshold value for a given feature and $i=$ $1,2, \ldots, N_{F}$, with $N_{F}$ being the total number of features used [34]. Features should be selected and calculated before using them in the DT to make the algorithm independent of the calculation cost of different 


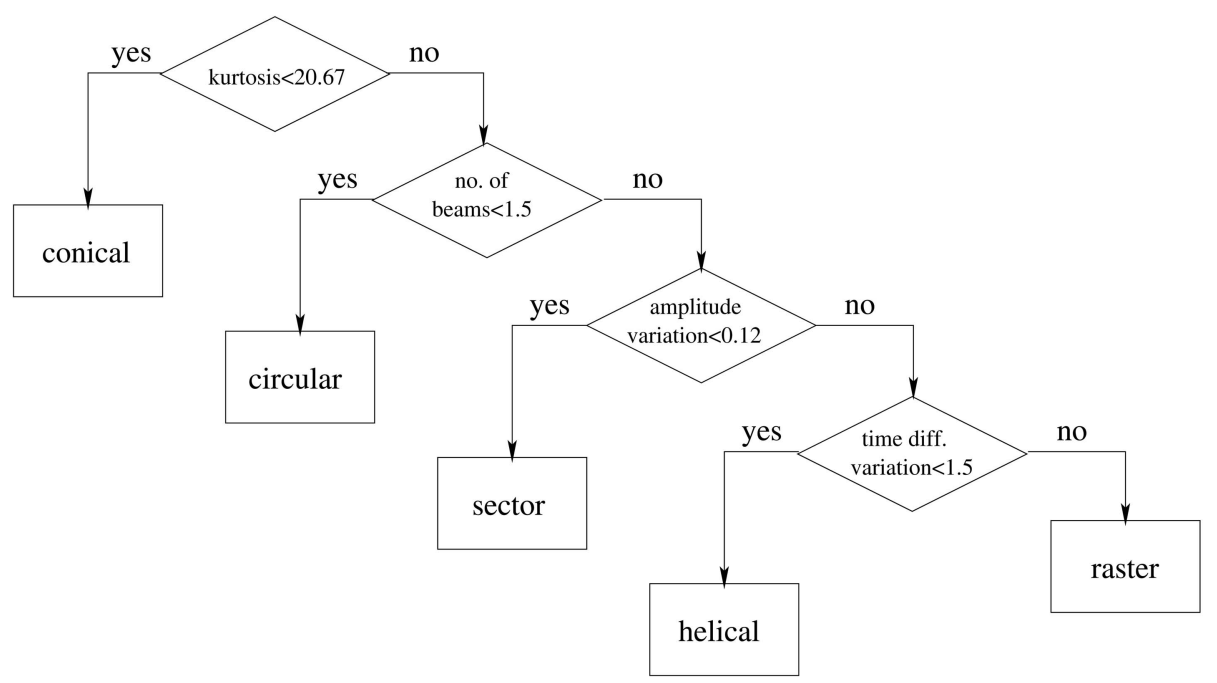

Fig. 12. BFTree DT classifier generated and used in this study.

features. These rules can be automatically extracted by different algorithms that examine the training vectors of all classes. More discriminative features are used at the nodes higher in the tree hierarchy. DT algorithms start at the top of the tree and branch out at each node into two descendant nodes based on checking conditions similar to the above. Given a set of training vectors along with their class labels, a binary tree, and a decision rule for each node of the tree, a particular subset of the training vectors is available at each node for classification. Each element of that subset satisfies the conditions imposed by the ancestors of that node. Thus, a decision at a node splits the corresponding subset into two: those that satisfy the condition and those that do not. Naturally, the ideal split is expected to isolate a class from others at each decision node. However, this is hardly the case in practice. This process continues until a terminating node (a leaf) is reached where the class of the input pattern (or the feature vector) is finally determined. After a complete tree is constructed, the tree size can be reduced by pruning by considering all pairs of neighboring leaf nodes. Pruning improves the efficiency and prevents overfitting. Test vectors are then used to evaluate the classification performance of the DT.

The important aspects of DT induction methods are, the number of splits at a node, splitting criterion, and the stopping criterion. As the information necessary to differentiate between the patterns is completely embodied in the decision rules, DTs have the advantage of not requiring storage of any reference feature vectors. DTs are nonmetric classifiers that do not rely on any measure of distance so that they are efficiently adapted to tasks where nominal features appear. However, real-valued features can also be used in the classification process.

There are a number of different tree growing algorithms that use training data to generate a DT to classify unknown patterns. The most commonly used
DT algorithms are classification and regression trees (CART) [35], C4.5 algorithm, J48 algorithm, and NB trees [33]. A large number of DTs can be combined to create an ensemble classifier called a random forest. A recently proposed best first tree (BFTree) learning algorithm [36] is considered in this study and found to classify the scans more accurately. The BFTree DT classifier generated and used in this study is illustrated in Fig. 12. The structure of our problem fits well into a DT-like structure since each of the selected features is discriminative for one or two of the scan types. For example, if the kurtosis value is low, than it is highly probable that AST is conical. If the number of main beams is two, AST is sectoral, etc.

3) Artificial Neural Networks: ANNs are classifiers that try to emulate the computational processes of the brain. They consist of nodes similar to the neurons in the nervous system. A smooth and nonlinear function, called the activation function, is used to model the process in the neuron. There are weighted connections between the nodes of the ANN that need to be adjusted through training. Different training algorithms can be used to compute the boundaries of decision regions in the form of connection weights and biases.

Multi-layer ANNs consist of an input layer, one or more hidden layers to extract progressively more meaningful features, and a single output layer, each comprised of a number of neurons. The input and output layers have sufficient numbers of nodes to cover the inputs and represent the outputs. The patterns are learned by the hidden layer(s) of the system. The number of hidden layers and the number of nodes in each hidden layer are chosen by the designer. If the number of neurons in the hidden layer(s) is too small, the ANN will not able to learn the patterns sufficiently well and the classification accuracy will not be satisfactory. However, an excess of neurons in the hidden layer(s) may complicate 
the training process and may lead to memorizing the signal instead of learning its dynamics. Due to the presence of distributed nonlinearity and a high degree of connectivity, theoretical analysis of ANNs is difficult. The performance of ANNs is affected by the choice of parameters related to the network structure, training algorithm, and input signals, as well as by parameter initialization [37].

There is a variety of network architectures, training algorithms, and activation functions for different applications [37]. In this study, the back-propagation algorithm [37] is used for training, by presenting a set of training patterns to the network. Back propagation is a supervised method that uses a gradient-descent procedure based on the error at the output. It tries to minimize the error by feeding the error at the output back to update the weights in each epoch. Different initial conditions and different numbers of neurons in the hidden layer have been considered. The aim is to minimize the average of the sum of squared errors over all training vectors:

$$
\mathcal{E}_{\mathrm{av}}(\mathbf{w})=\frac{1}{2 N_{p}} \sum_{i=1}^{N_{p}} \sum_{j=1}^{N_{c}}\left[d_{i j}-o_{i j}(\mathbf{w})\right]^{2} .
$$

Here, $\mathbf{w}$ is the weight vector, $d_{i j}$ and $o_{i j}$ are the desired and actual output values for the $i$ th training pattern and the $j$ th output neuron, and $N_{p}$ is the total number of training patterns. When the entire training set is covered, an epoch is completed. The error between the desired and actual outputs is computed at the end of each iteration and these errors are averaged at the end of each epoch (see (7)). The training process is terminated when a certain precision goal on the average error is reached or if the specified maximum number of epochs $(5,000)$ is exceeded, whichever occurs earlier. The latter case occurs very rarely. The acceptable average error level is set to a value of $5 \%$. The weights are initialized randomly with a uniform distribution in the interval $[0,1]$, and the learning rate is chosen as 0.05 .

After the network is trained, classification is performed. In the test phase, the test feature vectors are fed forward to the ANN, with the already converged weights, and the class of the signal is determined according to the output. The output neurons can take continuous values between 0 and 1 . The outputs are compared with the desired outputs, and the error between them is calculated. The test vector is said to be correctly classified if this error is below a threshold value of 0.15 .

In this work a fully connected three-layer ANN is used for classifying radar scan patterns. The input layer has as many neurons as the number of features used (or $N_{F}$, the dimension of the feature vectors), which is four here. The hidden layer has 10 neurons, and the output layer has five neurons, equal to the number of classes $N_{c}$. In the input and hidden layers each, there is an additional neuron with a bias value of one. For an input feature vector $\mathbf{z} \in \Re^{4}$, the desired output is one for the class that the vector belongs to, and zero for all other output neurons. The sigmoid function used as the activation function in the hidden and output layers is given by $g(x)=\left(1+e^{-x}\right)^{-1}$.

4) Support Vector Machines: SVMs are binary classifiers that try to partition the feature space with hyperplanes where each separated volume represents a different class [38]. This machine learning technique was first proposed early in the 1980s [39]. It has been used in applications such as object, voice, and handwritten character recognition, and text classification.

Consider a binary classification problem where $N_{p}$ training feature vectors $\mathbf{z}_{i}$ in some vector space $\mathcal{Z}$ and their binary class labels $\ell_{i} \in\{-1,1\}$ are available, where $\ell_{i}=\ell\left(\mathbf{z}_{i}\right)$ and $i=1, \ldots, N_{p}$. The goal in training an SVM is to find the hyperplane that maximizes the margin of separation between the classes so that the generalization of the classifier is better. All vectors lying on one side of the hyperplane (class 1 ) are labeled as $\ell_{i}=+1$, and all vectors lying on the other side (class 2) are labeled as $\ell_{i}=-1$. The support vectors are the training patterns that lie closest to the hyperplane and are at equal distance from it. They define the optimal separating hyperplane and are the most difficult patterns to classify, yet the most informative for the classification task.

If the feature vectors in the original feature space are not linearly separable, SVMs preprocess and represent them in a space of higher dimensionality where they become linearly separable. With a suitable nonlinear mapping $\varphi($.) to a sufficiently high dimension, data from two different classes can always be made linearly separable, and separated by a hyperplane. The choice of the nonlinear mapping depends on the prior information available to the designer. If such information is not available, one might choose to use polynomials, Gaussians, or other types of basis functions. The dimensionality of the transformed (mapped) space can sometimes be much higher than the original feature space.

The projection of the original training data in space $\mathcal{Z}$ to a higher dimensional feature space $\mathcal{F}$ is performed by using a Mercer kernel operator or kernel function $\mathcal{K}$ [40]. We consider a set of classifiers of the form $q(\mathbf{z})=\sum_{i=1}^{N_{p}} \beta_{i} \mathcal{K}\left(\mathbf{z}_{i}, \mathbf{z}\right)$ where $q(\mathbf{z})$ is a linear discriminant function in the transformed space. When $q(\mathbf{z}) \geq 0$, we label $\mathbf{z}$ as +1 , otherwise as -1 . When $\mathcal{K}$ satisfies Mercer's condition, $\mathcal{K}\left(\mathbf{z}_{i}, \mathbf{z}\right)=\varphi\left(\mathbf{z}_{i}\right) \cdot \varphi(\mathbf{z})$ where $\varphi():. \mathcal{Z} \rightarrow \mathcal{F}$ is a nonlinear mapping mentioned above and "." denotes the inner or dot product of two vectors. We can then rewrite $q(\mathbf{z})$ in the transformed space as $q(\mathbf{z})=\mathbf{a} \cdot \varphi(\mathbf{z})$, where $\mathbf{a}=\sum_{i=1}^{N_{p}} \beta_{i} \varphi\left(\mathbf{z}_{i}\right)$ is a weight vector. The separating hyperplane is $\mathbf{a} \cdot \varphi(\mathbf{z})=0$. (Here, both the weight vector $\mathbf{a}$ and the transformed feature vector $\varphi\left(\mathbf{z}_{i}\right)$ have been augmented 


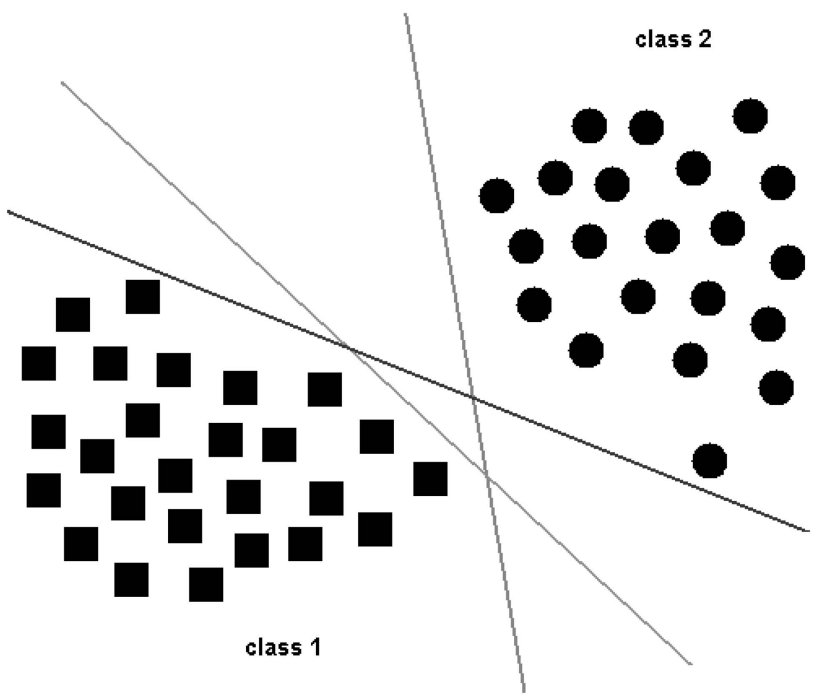

(a)

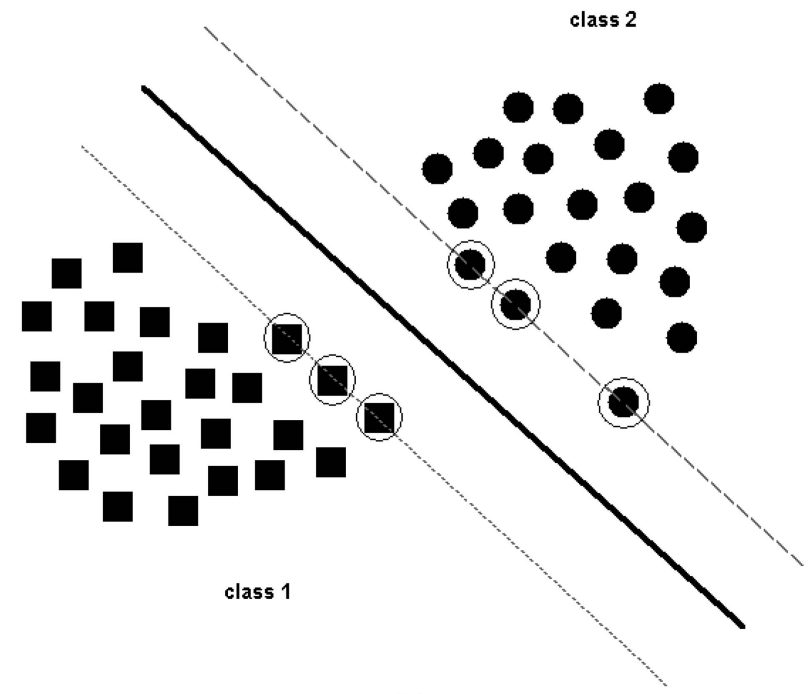

(b)

Fig. 13. (a) Three different hyperplanes separating two classes. (b) SVM hyperplane (solid line), its margins (dotted and dashed lines), and support vectors (circled solid squares and dots).

by one dimension to include a bias weight so that the hyperplanes need not pass through the origin.)

To illustrate the problem in 2-D, consider the training set feature vectors in Fig. 13. In this example, there are two classes; squares $\left(\ell_{i}=+1\right)$ symbolize the first class (class 1$)$ and solid dots $\left(\ell_{i}=-1\right)$ symbolize the second class (class 2). These two types of training vectors can be separated with infinitely many different hyperplanes, three of which are shown in Fig. 13(a). For each of these hyperplanes, correct classification rates may be different when test vectors are presented to the system. To have the smallest classification error at the test stage, the hyperplane should be placed between the support vectors of the two classes with maximum and equal margin for both classes. The optimal hyperplane $\mathbf{a} \cdot \varphi(\mathbf{z})=0$ is unique [34] and is represented by the solid line in Fig. 13(b). The equations of the margin lines that maximize the margin of separation between the support vectors are $\mathbf{a} \cdot \varphi(\mathbf{z})=1$ (shown in dotted lines) and $\mathbf{a} \cdot \varphi(\mathbf{z})=-1$ (shown in dashed lines), respectively. The support vectors are circled in the same figure. Because there should not be training set vectors dropping between these margin lines, the inequality $\ell_{i} q\left(\mathbf{z}_{i}\right)=$ $\ell_{i} \mathbf{a} \cdot \varphi\left(\mathbf{z}_{i}\right) \geq 1$ should be satisfied for $i=1, \ldots, N_{p}$.

In the next stage the $\beta_{i}$ s that correspond to the hyperplane in $\mathcal{F}$ with the maximal margin need to be computed. Maximizing the margin of separation is equivalent to minimizing $\frac{1}{2}\|\mathbf{a}\|^{2}$ subject to the constraint represented by the inequality given above [33]. Using the method of Lagrange multipliers, we construct the functional:

$$
\mathcal{L}(\mathbf{a}, \lambda)=\frac{1}{2}\|\mathbf{a}\|^{2}-\sum_{i=1}^{N_{p}} \lambda_{i}\left[\ell_{i} \mathbf{a} \cdot \varphi\left(\mathbf{z}_{i}\right)-1\right]
$$

where the second term in the equation expresses the goal of classifying the points correctly. The constrained optimization problem can be solved by using quadratic programming or other alternative techniques. To find the optimal hyperplane, we minimize $\mathcal{L}(\mathbf{a}, \lambda)$ with respect to the weight vector $\mathbf{a}$, while maximizing with respect to the undetermined Lagrange multipliers $\lambda_{i} \geq 0$. This is done by setting the partial derivatives of $\mathcal{L}(\mathbf{a}, \lambda)$ with respect to $\mathbf{a}$ and $\lambda_{i}$ to zero. Solving the resulting equations simultaneously will yield several non-zero $\lambda_{i}$. The $\mathbf{z}_{i}$ s that correspond to non-zero $\lambda_{i}$ will satisfy (8) and be the support vectors through which marginal hyperplanes shown in Fig. 13(b) will pass. The optimal solution of the weight vector is $\mathbf{a}^{*}=$ $\sum_{i=1}^{N_{p}} \ell_{i} \lambda_{i} \varphi\left(\mathbf{z}_{i}\right)$ corresponding to $\beta_{i}=\ell_{i} \lambda_{i}$. Then, the corresponding discriminant function is given by $q^{*}(\mathbf{z})=\sum_{i=1}^{N_{p}} \lambda_{i} \ell_{i} \varphi\left(\mathbf{z}_{i}\right) \cdot \varphi(\mathbf{z})$.

In this study the method described above is applied to differentiate five different radar ASTs. A linear SVM is trained with the available scan patterns without the use of any transformation to a higher dimensional space. In this relatively simpler case, $\varphi(\mathbf{z})$ and $\varphi\left(\mathbf{z}_{i}\right)$ in the above equations simplify to $\mathbf{z}$ and $\mathbf{z}_{i}$, respectively, and $\mathcal{K}\left(\mathbf{z}_{i}, \mathbf{z}\right)$ becomes $\mathbf{z}_{i} \cdot \mathbf{z}$, everything else being the same. Since there are more than two classes, one-versus-the-rest classification is performed and five different binary classifiers are trained, where each classifier recognizes one of five scan patterns.

\section{F. Results}

As mentioned above, 100 data sequences are generated by the ASPS; 20 from each of the basic ASTs considered in this study.

An open source java-based machine learning software, WEKA (Waikato Environment for 


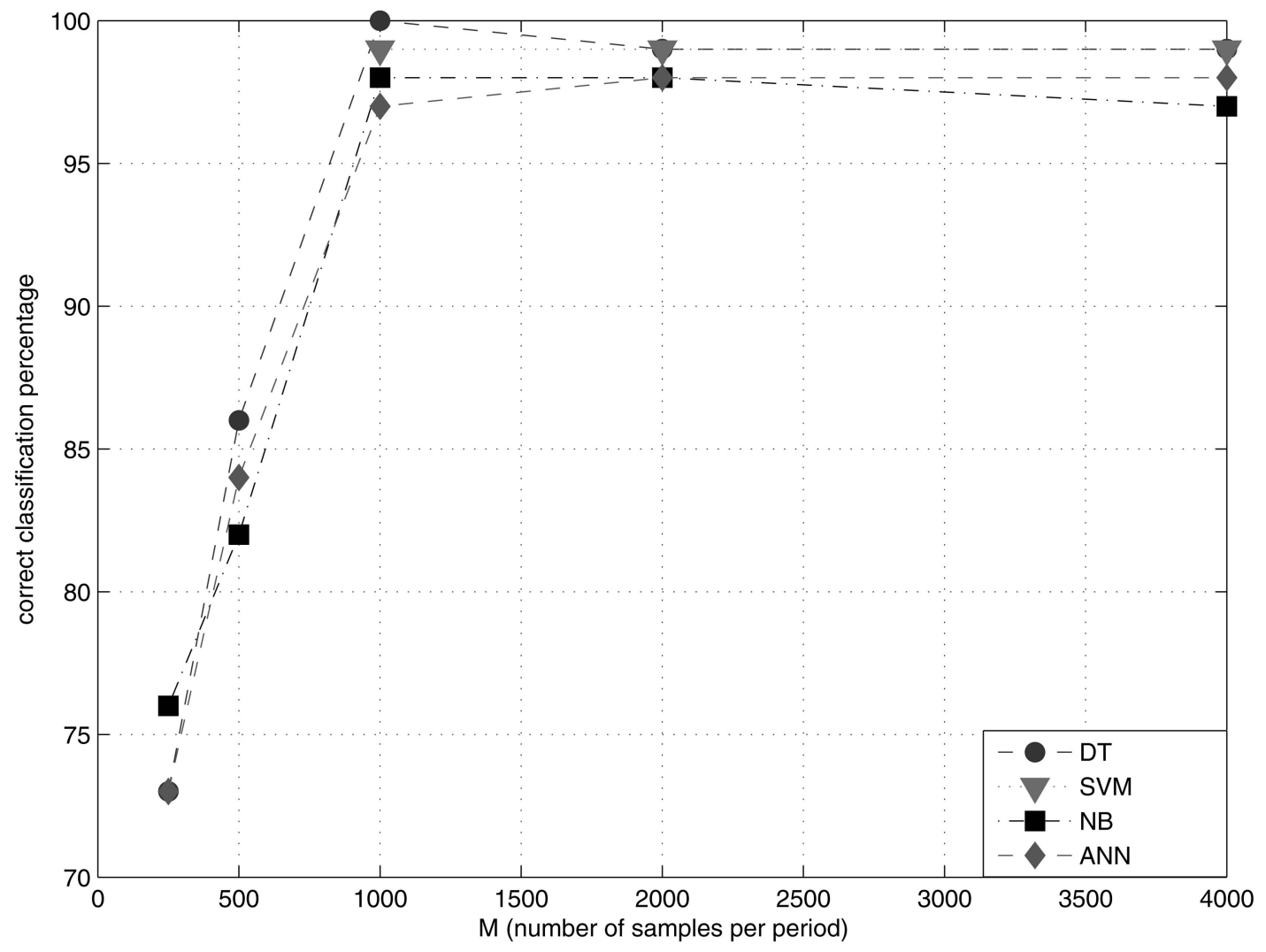

Fig. 14. Classification results of four classifiers as function of $M$.

Knowledge Analysis), developed by The University of Waikato, New Zealand, is used in the classification process [41]. We have used a four-fold crossvalidation technique for training and testing the algorithm where the feature vectors from each class are randomly partitioned into four. In each of the four runs, one of the partitions is retained for testing and the remaining three are used for training. This way, all of the feature vectors are tested [42].

Figure 14 illustrates the classification results with respect to different $M$ values for all four classifiers to see the effect of the number of samples per period. It can be observed that for small values of $M$, the signal is undersampled and the features are not correctly extracted, causing errors in the classification process. For SVM, the cases where $M<1,000$ did not converge. For $M \geq 1,000$, the classification percentage saturates around $99 \%$. One can conclude from the figure that $M=1,000$ is a good compromise between the classification accuracy and computational complexity. The confusion matrices of the classifiers for $M=1,000$ are given in Table II where the differentiation accuracy is equal or above 97\%. The DT classifier results in greater classification accuracy than the other classifiers.

The effect of noise is also considered and analyzed. Added noise affects the detected signal levels because of the thresholding process in the detection of the pulses. A threshold level $10 \mathrm{~dB}$
TABLE II

Confusion Matrices of the (a) NB, (b) DT, (c) ANN, (d) SVM Classifiers: $98 \%, 100 \%, 97 \%$, and $99 \%$ Correct Classification Rates are Achieved, Respectively

\begin{tabular}{|c|c|c|c|c|c|c|}
\hline & \multicolumn{5}{|c|}{ Classified } \\
\hline & & Circular & Sector & Raster & Helical & Conical \\
\hline \multirow[t]{5}{*}{ (a) true class } & circular & 19 & 1 & 0 & 0 & 0 \\
\hline & sector & 1 & 19 & 0 & 0 & 0 \\
\hline & raster & 0 & 0 & 20 & 0 & 0 \\
\hline & helical & 0 & 0 & 0 & 20 & 0 \\
\hline & conical & 0 & 0 & 0 & 0 & 20 \\
\hline \multirow[t]{5}{*}{ (b) true class } & circular & 20 & 0 & 0 & 0 & 0 \\
\hline & sector & 0 & 20 & 0 & 0 & 0 \\
\hline & raster & 0 & 0 & 20 & 0 & 0 \\
\hline & helical & 0 & 0 & 0 & 20 & 0 \\
\hline & conical & 0 & 0 & 0 & 0 & 20 \\
\hline \multirow[t]{5}{*}{ (c) true class } & circular & 20 & 0 & 0 & 0 & 0 \\
\hline & sector & 0 & 19 & 1 & 0 & 0 \\
\hline & raster & 0 & 2 & 18 & 0 & 0 \\
\hline & helical & 0 & 0 & 0 & 20 & 0 \\
\hline & conical & 0 & 0 & 0 & 0 & 20 \\
\hline \multirow[t]{5}{*}{ (d) true class } & circular & 20 & 0 & 0 & 0 & 0 \\
\hline & sector & 0 & 19 & 0 & 1 & 0 \\
\hline & raster & 0 & 0 & 20 & 0 & 0 \\
\hline & helical & 0 & 0 & 0 & 20 & 0 \\
\hline & conical & 0 & 0 & 0 & 0 & 20 \\
\hline
\end{tabular}

above the noise power level is used in this analysis. A similar threshold level is usually used in EW systems as a good compromise between the probability of 


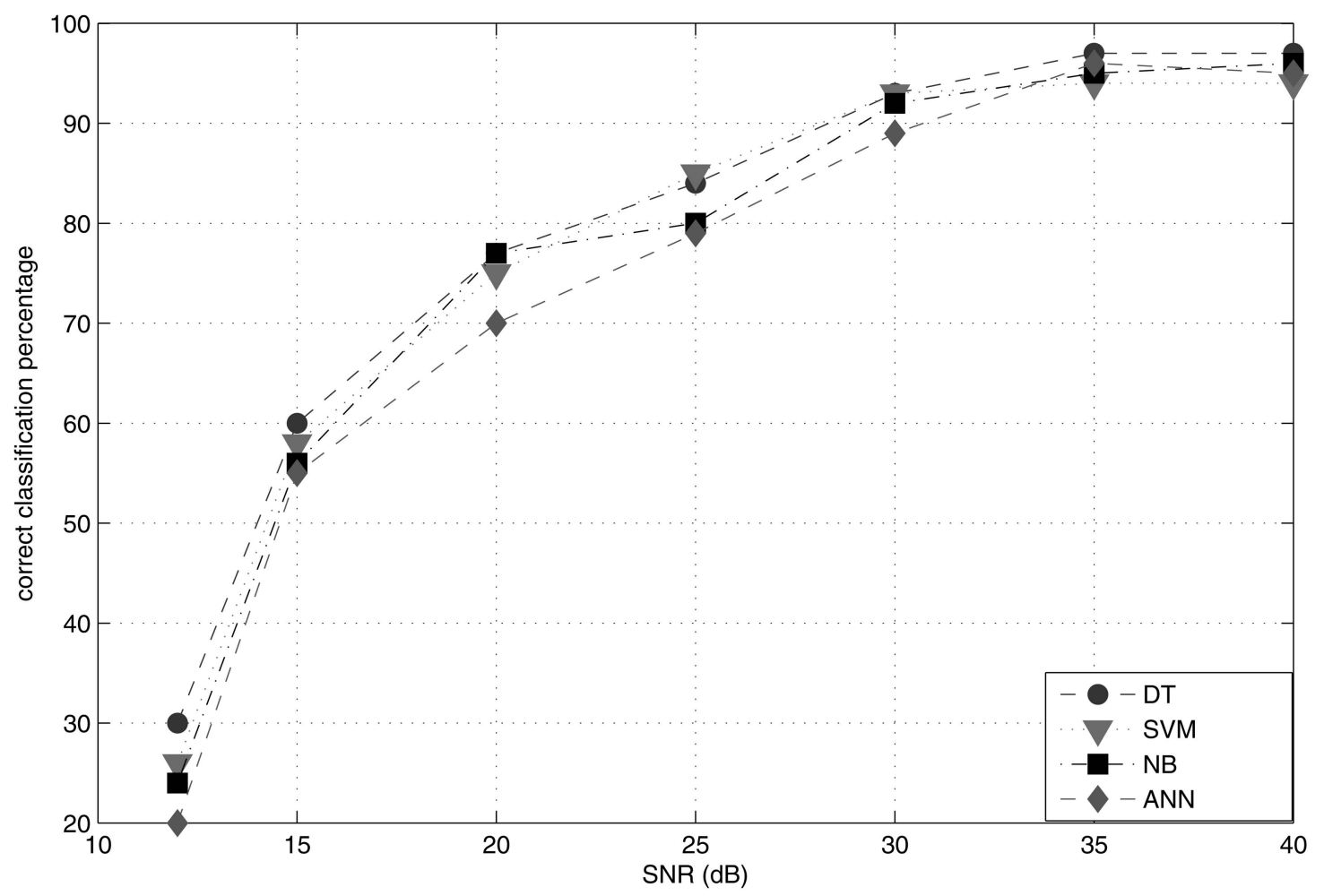

Fig. 15. Classification results of four classifiers for different SNRs.

detection and the probability of false alarm [4].

Thresholding limits the range of the PA versus ToA signal. This, of course, may result in the loss of the mainlobes, especially in the raster and helical scans, leading to classification errors. For example, because of the limited amplitude range of the signal, only one bar of the raster scan may be observed, which leads to an erroneous circular scan classification. Classification results with changing signal-to-noise ratios (SNRs) are shown in Fig. 15; we used SNR values of 12, 15, 20, $25,30,35$, and $40 \mathrm{~dB}$. The breakdown in performance around $25 \mathrm{~dB}$ SNR is caused by the limited amplitude range of the signal, discussed earlier. This limited range indicates a tendency towards sector and circular scans both of which are azimuth-only scans and relatively immune to the range of the received signal. We have also observed that when the sinusoidal shape in a conical scan is distorted by the loss of pulses, the algorithm again has a tendency towards a circular scan. The results also indicate that ANN is less robust to noise compared with the other classifiers. The confusion matrices of all four classifiers for the $20 \mathrm{~dB}$ SNR case are given in Table III.

The analysis has also shown that the noise level of the EW receiver greatly affects the classification performance; the signal's amplitude range decreases with increasing noise level. The amplitude range of the signal is very important, especially for ASTs that scan both in azimuth and elevation (raster and helical). The range limitation of the signal results in information loss (main beams with different elevation
TABLE III

Confusion Matrices of the (a) NB, (b) DT, (c) ANN, (d) SVM classifiers for $20 \mathrm{~dB}$ SNR: $77 \%, 77 \%, 70 \%$, and $75 \%$ Correct Classification Rates are Achieved, Respectively

\begin{tabular}{lc|ccccc}
\hline \hline & & \multicolumn{5}{|c}{ Classified } \\
& & Circular & Sector & Raster & Helical & Conical \\
\hline (a) true class & circular & 19 & 1 & 0 & 0 & 0 \\
& sector & 1 & 19 & 0 & 0 & 0 \\
& raster & 6 & 0 & 4 & 10 & 0 \\
& helical & 0 & 5 & 0 & 15 & 0 \\
& conical & 2 & 0 & 0 & 0 & 18 \\
\hline (b) true class & circular & 20 & 0 & 0 & 0 & 0 \\
& sector & 1 & 15 & 0 & 4 & 0 \\
& raster & 7 & 0 & 8 & 5 & 0 \\
& helical & 3 & 3 & 0 & 14 & 0 \\
& conical & 0 & 0 & 0 & 0 & 20 \\
\hline (c) true class & circular & 20 & 0 & 0 & 0 & 0 \\
& sector & 0 & 18 & 0 & 2 & 0 \\
& raster & 8 & 1 & 6 & 5 & 0 \\
& helical & 3 & 3 & 6 & 8 & 0 \\
& conical & 2 & 0 & 0 & 0 & 18 \\
\hline (d) true class & circular & 20 & 0 & 0 & 0 & 0 \\
& sector & 0 & 18 & 0 & 2 & 0 \\
& raster & 7 & 3 & 6 & 4 & 0 \\
& helical & 3 & 4 & 0 & 13 & 0 \\
& conical & 2 & 0 & 0 & 0 & 18 \\
\hline & & & & & &
\end{tabular}

levels are not sensed in the EW receiver); it cannot be inferred in any other way because of the detection schemes of the EW receivers. The classification accuracy of raster and helical scans degrades significantly with the signal's range limitation. 
TABLE IV

Time Needed for Training and Testing Each Classifier

\begin{tabular}{ccccc}
\hline \hline classifier & NB & DT & ANN & SVM \\
\hline time $(\mathrm{s})$ & 0.08 & 0.05 & 0.35 & 0.12 \\
\hline
\end{tabular}

Circular, conical, and sector scans are more robust to this effect. To make the algorithm more reliable, a simple check of amplitude range can be included before the algorithm is executed. A range greater than $15 \mathrm{~dB}(\mathrm{SNR} \geq 25 \mathrm{~dB})$ ensures classification accuracies above $90 \%$.

1) Comparison of the Computational Time of the Classifiers: To determine the computational complexity of the classifiers considered in this study, we analyzed the time it takes to train and test each classifier. A computer with a $2.66 \mathrm{GHz}$ processor and 3 GB of RAM was used for this purpose. The results, obtained with WEKA and given in Table IV, indicate that the DT classifier outperforms the other classifiers in this aspect as well.

2) Validation with Real Signals: ASELSAN Inc. [43] acquired real signals from different pulsed radar with its own EW receivers configured on a moving airborne platform. The recorded signals were PRI modulated but intrapulse modulation was not used. ASELSAN Inc. provided the data for validation purposes only; its description and illustration were strictly forbidden because of the classification level. Since the EW platform was moving, the assumption of stationarity of the EW receiver (Section II) was not met, making the classification process even more challenging. The signal also had some outliers caused by the movement of and reflections from the platform. These effects resulted in distortions in the main beam.

The algorithm had to be modified because of the properties of the signal. The threshold level in period estimation was decreased to 0.85 because of the effect of non-Gaussian noise. It was not possible to coherently average $K$ periods of the signal because the motion of the platform shifted the instant the mainlobe was detected. Thus, instead of averaging all the available periods in the signal, we chose the largest main beam and the one-period interval around it for feature extraction.

A total of 30 signal vectors were acquired where the SNR varies between $20-35 \mathrm{~dB}$. The real data is comprised of circular, conical, and sector scans. The DT classifier is used because of its high classification accuracy and computational simplicity. The confusion matrix for the real data is given in Table V. The classification error rate of the algorithm is 1 in 30 data sequences in this case. The only error seen is a sector-raster misclassification, which is expected because of the similar nature of these scans. The results indicate that the algorithm presented in this study can handle real signals that have outlier-like
TABLE V

Confusion Matrix for Real Signals (97\% Correct Classification)

\begin{tabular}{c|ccccc}
\hline \hline \multirow{2}{*}{ True Class } & Circular & Sector & Raster & Helical & Conical \\
\hline circular & 10 & 0 & 0 & 0 & 0 \\
\hline sector & 0 & 9 & 1 & 0 & 0 \\
\hline conical & 0 & 0 & 0 & 0 & 10 \\
\hline
\end{tabular}

shot noises and deformed main beams. The validation phase of the algorithm ensured that it is robust against probable complications encountered in the real world.

\section{CONCLUSIONS}

We addressed the problem of radar ASP estimation and AST classification in EW signal processing on which no clear and accessible study is available in the open literature. The research for an automatized solution to the problem led to a sufficiently general algorithm that uses the conventional parameters of radar systems. The main contribution of this work is the design of a novel and robust algorithm for AST classification. Unlike the two patents that used correlation-based techniques for AST recognition, our approach is based on ASP estimation in the time domain, preprocessing, feature extraction, and pattern classification.

The algorithm is validated by both synthetic data generated by the ASPS and a limited amount of real data acquired and provided by ASELSAN Inc. Classification accuracy above $97 \%$ is achieved in most cases, with the DT classifier resulting in the highest values. The robustness of this classifier to noise and its low computational complexity make it very useful for this particular application.

Although the algorithm in this study has been developed and tested for conventional pulsed radar systems with and without modulation, it is equally applicable to continuous-wave (CW) radar systems since the relevant parameters of $\mathrm{CW}$ radars can be obtained by chopping up the $\mathrm{CW}$ signal into small pulses. The proposed algorithm framework can be used not only for the particular problem addressed here, but also to analyze signals in applications where uniformly or nonuniformly sampled periodic signals need to be classified. One example is vowel classification in speech recognition. The parameters of the algorithm such as the threshold levels or the value of $M$ should be chosen according to the application at hand.

Our future work involves modifying the algorithm so that it can operate in real time as the radar signal is acquired by an EW receiver. The main difficulty here would be to differentiate the sidelobes from the mainlobe when only partial data is observed instead of the whole. Other possible research directions would be handling the changing parameters of a particular 
emitter (e.g., frequency hopping and frequency agility), sudden transitions between different ASTs, handling multiple radar signals from different emitters, and antenna scan analysis of phased-array radars.

\section{REFERENCES}

[1] Joint Staff

Joint Publication 3-13.1 Electronic Warfare.

United States Army Joint Publication, Jan. 25, 2007.

[2] Neri, F

Introduction to Electronic Defense Systems (2nd ed.).

Norwood, MA: Artech House, 2001.

[3] Wikipedia http://en.wikpedia.org/wiki/Electronic_warfare.

[4] Wiley, R. G.

ELINT: The Interception and Analysis of Radar Signals. Norwood, MA: Artech House, 2006.

[5] Zaczek, T. E.

Antenna scan pattern generator.

U.S. Patent 4,327,417, 1980.

[6] Greer, T. H.

Automatic recognition of radar scan type.

U.S. Patent 6,697,007, 2004.

[7] Keogh, E. and Ratanamahatana, C. A.

Exact indexing of dynamic time warping.

Knowledge and Information Systems, 7, 3 (2005), 358-386.

[8] Deller, Jr., J. R., Hansen, J. H. L., and Proakis, J. G.

Discrete-Time Processing of Speech Signals.

Hoboken, NJ: Wiley-IEEE Press, 1999, ch.11, pp. 623-674.

[9] Keogh, E. and Kasetty, S.

On the need for time series data mining benchmarks: A survey and empirical demonstration.

Data Mining and Knowledge Discovery, 7, 4 (2003), 349371.

[10] Agrawal, R., Faloutsos, C., and Swami, A.

Efficient similarity search in sequence databases.

Foundations of Data Organization and Algorithms, Lecture

Notes in Computer Science, vol. 730, 1993, pp. 69-84.

[11] Agrawal, R., et al.

Fast similarity search in the presence of noise, scaling, and translation in time-series databases.

Proceedings of the 21st International Conference on Very

Large Data Bases, Zurich, Switzerland, Sept. 11-15, 1995.

[12] Chan, K. P. and Fu, A. W-C.

Efficient time series matching by wavelets.

In Proceedings of the 15th International Conference on

Data Engineering, Sydney, Australia, Mar. 23-26, 1999,

p. 126.

[13] Wu, Y-L., Agrawal, D., and El Abbadi, A.

A comparison of DFT and DWT based similarity search

in time-series databases.

In Proceedings of the Ninth International Conference on Information and Knowledge Management, McLean, VA, Nov. 6-11, 2000, pp. 488-495.

[14] Hmam, H

Scan-based emitter passive localization.

IEEE Transactions on Aerospace and Electronic Systems,

43, 1 (Jan. 2007), 36-54.

[15] Doğançay, K.

Online optimization of receiver trajectories for scan-based emitter localization.

IEEE Transactions on Aerospace and Electronic Systems,

43, 3 (July 2007), 1117-1125.

[16] Hmam, H. and Doğançay, K.

Passive localization of scanning emitters.

IEEE Transactions on Aerospace and Electronic Systems,

46, 2 (Apr. 2010), 944-951.
[17] Mahafza, B. R.

Radar Systems Analysis and Design Using MATLAB.

Boca Raton, FL: Chapman \& Hall/CRC Press, 2000.

[18] Skolnik, M. I.

Introduction to Radar Systems (3rd ed.).

New York: McGraw-Hill, 2000.

[19] Skolnik, M. I. (Ed.)

Radar Handbook, (3rd ed.).

New York: McGraw-Hill, 2008.

[20] United States Navy

Electronic Warfare and Radar Systems Engineering Handbook.

Naval Air Systems Command, Avionics Dept.,

Washington, D.C., 1999.

[21] Balanis, C. A.

Antenna Theory Analysis and Design (3rd ed.).

Hoboken, NJ: Wiley, 1997.

[22] Stimson, G. W.

Introduction to Airborne Radar (2nd ed.).

Raleigh, NC: SciTech Publishing, 1998.

[23] Adamy, D. L.

Introduction to Electronic Warfare: Modeling and Simulation.

Raleigh, NC: SciTech Publishing Inc., 2006.

[24] Adamy, D.

EW 101: A First Course in Electronic Warfare.

Norwood, MA: Artech House, 2001.

[25] Nickel, U.

Fundamentals of signal processing for phased array radar.

In Advanced Radar Signal and Data Processing,

Educational Notes RTO-EN-SET-086, Paper 1, pp.

1-1-1-22. Neuilly-sur-Seine, France: NATO Research \&

Technology Organisation (RTO), 2006.

[26] Eravc1, B.

Automatic radar antenna scan analysis in electronic warfare

Master's thesis, Bilkent University, Dept. of Electrical and Electronics Engineering, Ankara, Turkey, Aug. 2010.

[27] Rabiner, L., et al.

A comparative performance study of several pitch detection algorithms.

IEEE Transactions on Acoustics, Speech, and Signal

Processing, ASSP-24, 5 (Oct. 1976), 399-418.

[28] Cheng, Y. M. and O'Shaughnessy, D.

Automatic and reliable estimation of glottal closure instant and period.

IEEE Transactions on Acoustics, Speech, and Signal

Processing, 37, 12 (Dec. 1989), 1805-1815.

[29] de Cheveign, A. and Kawahara, H.

Multiple period estimation and pitch perception model.

Speech Communication, 27, 3-4 (Apr. 1999), 175-185.

[30] de Cheveign, A. and Kawahara, $\mathrm{H}$.

YIN, a fundamental frequency estimator for speech and music.

Journal of the Acoustical Society of America, 111, 4 (Apr. 2002), 1917-1930.

[31] Resch, B., et al.

Estimation of the instantaneous pitch of speech.

IEEE Transactions on Audio, Speech, and Language

Processing, 15, 3 (Mar. 2007), 813-822.

[32] Crochiere, R. E. and Rabiner, L. R.

Multirate Digital Signal Processing.

Upper Saddle River, NJ: Prentice-Hall, 1983.

[33] Duda, R. O., Hart, P. E., and Stork, D. G.

Pattern Classification (2nd ed.).

Hoboken, NJ: Wiley, 2001.

[34] Theodoridis, S. and Koutroumbas, K.

Pattern Recognition (4th ed.).

Burlington, MA: Academic Press, 2009. 
Breiman, L., et al.

Classification and Regression Trees.

Boca Raton, FL: Chapman \& Hall/CRC, 1998.

Shi, H.

Best-first decision tree learning.

Master's thesis, University of Waikato, Hamilton, New Zealand, 2006.

Haykin, S.

Neural Networks and Learning Machines (3rd ed.). Upper Saddle River, NJ: Prentice-Hall, 2009.

Shawe-Taylor, J. and Cristianini, N.

An Introduction to Support Vector Machines and Other Kernel-Based Learning Methods.

New York: Cambridge University Press, 2000.

Vapnik, V. N.

Estimation of Dependences Based on Empirical Data. Moscow, Russia: Nauka, 1979 (in Russian), English translation: New York: Springer-Verlag, 1982.
[40]

Tong, S. and Koller, D.

Support vector machine active learning with applications to text classification.

Journal of Machine Learning Research, 2 (Nov. 2001), 45-66.

[41] Hall, M., et al.

The WEKA data mining software: An update. ACM SIGKDD Explorations Newsletter, 11, 1 (June 2009), 10-18, http://www.cs.waikato.ac.nz/ml/weka/.

[42] Witten, I. H. and Frank, E.

Data Mining: Practical Machine Learning Tools and Techniques (2nd ed.).

San Francisco, CA: Morgan Kaufmann, 2005.

ASELSAN Inc.

Radar, Electronic Warfare, and Intelligence Systems Division (REHIS), Yenimahalle, 06370, Ankara, Turkey, http://www.aselsan.com.tr.
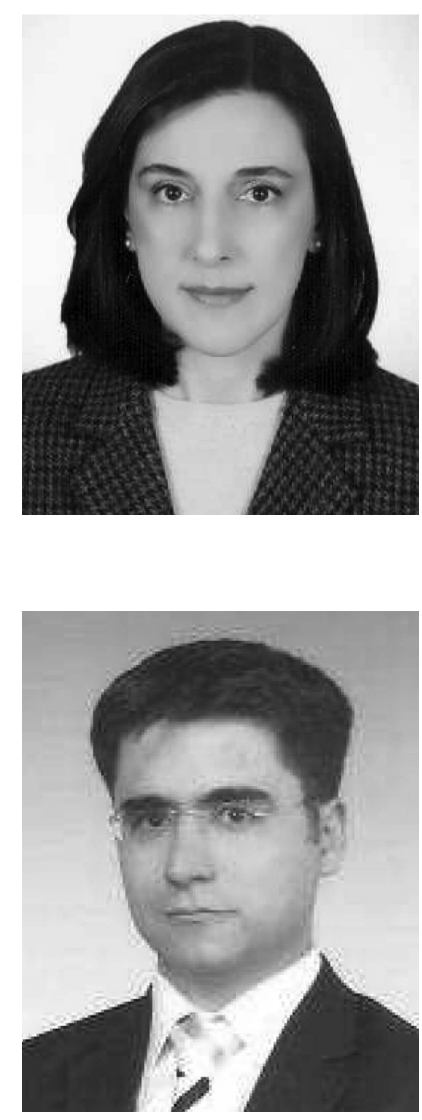

Billur Barshan received B.S. degrees in both electrical engineering and physics from Boğaziçi University in Istanbul, Turkey in 1986 and her M.S. and Ph.D. degrees in electrical engineering from Yale University, New Haven, CT, in 1988 and 1991, respectively.

She was a research assistant at Yale from 1987 to 1991 and a postdoctoral researcher at the Robotics Research Group at the University of Oxford, from 1991 to 1993. In 1993, she joined the faculty of Bilkent University in Ankara, Turkey, where she is a professor in the Department of Electrical and Electronics Engineering. She is also the founder of the Robotics and Sensing Laboratory in the same department. Her current research interests include sensor-based robotics, intelligent sensing, ultrasonic, optical, and inertial sensing, sensor signal processing, multi-sensor data fusion, and human activity recognition and classification.

Dr. Barshan is the recipient of the 1994 Nakamura Prize for the most outstanding paper at the 1993 IEEE/RSJ Intelligent Robots and Systems International Conference, the 1998 TÜBITAK Young Investigator Award, and the 1999 Mustafa N. Parlar Foundation Research Award.

Bahaeddin Eravcl received his B.S. and M.S. degrees in electrical and electronics engineering from Hacettepe University and Bilkent University, Ankara, Turkey in 2007 and 2010, respectively.

He is currently employed by ASELSAN Inc., in Ankara and is working towards his Ph.D. degree in computer science at Bilkent University. Among his research interests are signal processing, machine learning, and data mining. 